\title{
On a new approach to the analysis of variance for experiments with orthogonal block structure. IV. Experiments in split-plot designs

\author{
Tadeusz Caliński, Agnieszka Łacka, Idzi Siatkowski
}

\author{
Department of Mathematical and Statistical Methods, \\ Poznań University of Life Sciences, Wojska Polskiego 28, 60-637 Poznań, Poland, \\ e-mail: calinski@up.poznan.pl, agnieszka.lacka@up.poznan.pl, \\ idzi.siatkowski@up.poznan.pl
}

\section{SUMMARY}

This paper provides estimation and hypothesis testing procedures for experiments in split-plot designs. These experiments have been shown to have a convenient orthogonal block structure when properly randomized. Due to this property, the analysis of experimental data can be carried out in a relatively simple manner. Relevant simplification procedures are indicated. According to the adopted approach, the analysis of variance and hypothesis testing procedures can be performed directly, rather than by combining the results of analyses based on some stratum submodels. The practical application of the presented theory is illustrated by examples of real experiments in appropriate split-plot designs. The present paper is the fourth in the planned series of publications on the analysis of experiments with orthogonal block structure.

Key words: analysis of variance, estimation, hypothesis testing, orthogonal block structure, randomization-derived model, split-plot designs

\section{Introduction}

The concept of orthogonal block structure is a desirable property. It was originally considered for a wide class of designs by Nelder (1965) and then formalized by Houtman and Speed (1983, Section 2.2). Due to them, the following definition of this concept can be adopted.

Definition 1.1. An experiment is said to have the orthogonal block structure (OBS) if the covariance (dispersion) matrix of the random variables 
observed on the experimental units (plots), $\boldsymbol{y}=\left[y_{1}, y_{2}, \ldots, y_{n}\right]^{\prime}$, has a representation of the form

$$
\mathrm{D}(\boldsymbol{y})=\sigma_{1}^{2} \phi_{1}+\sigma_{2}^{2} \phi_{2}+\cdots+\sigma_{t}^{2} \phi_{t},
$$

where the $\left\{\phi_{\alpha}\right\}, \alpha=1,2, \ldots, t$, are known symmetric, idempotent and pairwise orthogonal matrices, summing to the identity matrix, the last usually being of the form $\phi_{t}=n^{-1} \mathbf{1}_{n} \mathbf{1}_{n}^{\prime}$.

It is interesting that experiments having the OBS property can be analyzed in a comparatively simple way. In particular, the analysis of variance (ANOVA) can be performed directly, not according to the classical procedure (initiated by Yates, 1939, 1940) of first conducting the analyses based on stratum submodels, and then combining their results (as can be seen in most of the relevant literature; see, e.g., Kala, 2019).

The analytical advantage of the OBS property is common to all experiments conducted in designs inducing this property. But details in performing the analysis of experimental data depend on the structure of the applied experimental design, making it advisable to present the relevant analytical methods for different classes of designs separately. Thus, it has been decided to publish a series of papers focused on practical applications of the new approach to the analysis of experiments with the OBS property. The first (Caliński and Siatkowski, 2017) is devoted to experiments conducted in proper block designs. The second (Caliński and Siatkowski, 2018) is devoted to experiments in nested block designs. The third (Caliński, Łacka and Siatkowski, 2019) concerns experiments in row-column designs. In each of these papers the subject is confined to experiments with the desirable OBS property. The present paper is devoted to experiments conducted in split-plot designs inducing the OBS property.

Split-plot designs are often used in practice, mainly in factorial experiments. The split-plot type designs are applied in situations where for a factorial experiment different types of experimental units are used, and where the levels of some factors are applied sequentially with separate randomization procedures, as noted by Hinkelmann and Kempthorne (2008, p. 533). Usually, particularly in agricultural experimentation, this type of design is used in two-factor experiments. Such a simple split-plot design was introduced by Yates $(1933,1935)$. Two-factor experiments in incomplete block designs with split-plots have been extensively described and illustrated by S. Mejza (1986). A review of the extensive literature on this subject is given there. More recently this type of design has been described and illustrated 
by examples in Bailey (2008, Chapter 8 ). That chapter is devoted to the concept of "Experimental units bigger than observation units". This particularly applies to the structure of split-plot designs, considered in Section 8.3 of Bailey's aforementioned book. But note that this concerns nested block designs also. At this point it may be interesting to note that Example 1 in Caliński and Siatkowski (2018) illustrates a split-plot design considered by Brzeskwiniewicz (1994).

The purpose of the present paper is to show how the OBS property of an experiment in a split-plot design provides the possibility of performing the analysis of experimental data with a comparatively simple methodology. Similarly as in the three previous papers of the present series, Section 2 of this paper is devoted to presentation of the randomization-derived mixed model, from which the described methodology follows. The theoretical background of the derived analysis is presented in Section 3. Then, in Section 4 , some simplifications of the proposed analytical methods are suggested. In Section 5 attention is drawn to some consequences following from the use of estimated stratum variances. Some examples illustrating the application of the derived analytical methods, ANOVA in particular, are presented in Section 6. Several concluding remarks concerning the advantages of the proposed approach are given in Section 7.

\section{A randomization-derived model}

Consider an experiment conducted in a split-plot design with $v$ treatments (the $v=v_{A} v_{B}$ treatments being the combined levels of a $v_{A} \times v_{B}$ factorial experiment) allocated in $b$ blocks, each composed of $k_{1}$ units (plots). Each of these main plots is subdivided into $k_{2}$ sub-plots (also called split-plots). Such a split-plot design is justifiably said to induce the OBS property (in the above sense).

Suppose that independent randomizations of blocks, of main plots within the blocks and of sub-plots within the main plots have been implemented in the considered experiment according to the usual procedure (following Nelder, 1965). The randomization-derived model can then be written as

$$
\boldsymbol{y}=\boldsymbol{X}_{1} \boldsymbol{\tau}+\boldsymbol{X}_{2} \boldsymbol{\beta}+\boldsymbol{X}_{3} \boldsymbol{\gamma}+\boldsymbol{\eta}+\boldsymbol{e},
$$

where $\boldsymbol{y}$ is an $n \times 1$ vector of $n=b k_{1} k_{2}$ observable data (lexicographically ordered with respect to blocks, main plots and sub-plots) concerning yield (or another variable trait) of interest in the analyzed experiment. As to the 
right side in (1), note that $\boldsymbol{X}_{1}$ is the $n \times v$ design matrix for treatment combinations, their unobservable parameters (fixed effects) being represented by $\boldsymbol{\tau}=\left[\tau_{1}, \tau_{2}, \ldots, \tau_{v}\right]^{\prime}, \boldsymbol{X}_{2}$ is the $n \times b$ design matrix for blocks, their random effects being represented by $\boldsymbol{\beta}=\left[\beta_{1}, \beta_{2}, \ldots, \beta_{b}\right]^{\prime}, \boldsymbol{X}_{3}$ is the $n \times\left(b k_{1}\right)$ design matrix for main plots, their random effects being represented by $\gamma=$ $\left[\gamma_{1}^{\prime}, \gamma_{2}^{\prime}, \ldots, \gamma_{b}^{\prime}\right]^{\prime}$, with $\gamma_{h}=\left[\gamma_{1(h)}, \gamma_{2(h)}, \ldots, \gamma_{k_{1}(h)}\right]^{\prime}$, for $h=1,2, \ldots, b$, while the $n \times 1$ vectors $\boldsymbol{\eta}$ and $\boldsymbol{e}$ stand for the sub-plot error and technical error random variables, all of these random variables being unobservable.

Note that the design matrices in (1) can be written more precisely as

$$
\begin{aligned}
& \boldsymbol{X}_{1}=\left[\boldsymbol{X}_{11}^{\prime}: \boldsymbol{X}_{12}^{\prime}: \cdots: \boldsymbol{X}_{1 b}^{\prime}\right]^{\prime}, \text { with } \boldsymbol{X}_{1 h}=\left[\boldsymbol{X}_{1 h 1}^{\prime}: \cdots: \boldsymbol{X}_{1 h k_{1}}^{\prime}\right]^{\prime}, \\
& \boldsymbol{X}_{2}=\boldsymbol{I}_{b} \otimes \mathbf{1}_{k_{1}} \otimes \mathbf{1}_{k_{2}} \text { and } \boldsymbol{X}_{3}=\boldsymbol{I}_{b} \otimes \boldsymbol{I}_{k_{1}} \otimes \mathbf{1}_{k_{2}},
\end{aligned}
$$

the last two with the use of Kronecker's product, denoted by $\otimes$.

The whole design composed of the main plots (the main plot design), denoted by $\mathcal{D}^{*}$, can be described by the $v \times\left(b k_{1}\right)$ incidence matrix

$$
\boldsymbol{N}=\boldsymbol{X}_{1}^{\prime} \boldsymbol{X}_{3}=\left[\boldsymbol{N}_{1}: \boldsymbol{N}_{2}: \cdots: \boldsymbol{N}_{b}\right],
$$

with $\boldsymbol{N}_{h}=\boldsymbol{X}_{1 h}^{\prime} \boldsymbol{X}_{3 h}=\boldsymbol{X}_{1 h}^{\prime}\left(\boldsymbol{I}_{k_{1}} \otimes \mathbf{1}_{k_{2}}\right)$ as the $v \times k_{1}$ incidence matrix describing the $h$ th component design (i.e., for the $h$ th block), denoted by $\mathcal{D}_{h}$, where $\boldsymbol{N}_{h}^{\prime} \mathbf{1}_{v}=k_{2} \mathbf{1}_{k_{1}}$ and $\boldsymbol{N}_{h} \mathbf{1}_{k_{1}}=\boldsymbol{r}_{h}$, the vector of treatment replications in $\mathcal{D}_{h}, h=1,2, \ldots, b$. Furthermore, it can be noted that the design, denoted by $\mathcal{D}$, according to which the $v$ treatments are assigned to the $b$ blocks is described by the $v \times b$ incidence matrix

$$
\boldsymbol{M}=\boldsymbol{X}_{1}^{\prime} \boldsymbol{X}_{2}=\left[\boldsymbol{r}_{1}: \boldsymbol{r}_{2}: \cdots: \boldsymbol{r}_{b}\right],
$$

from which $M 1_{b}=r$, the vector of treatment replications in the whole design.

Because both $\mathcal{D}^{*}$ and $\mathcal{D}$ are proper, an experiment in such a split-plot design has the OBS property. This allows the model to be resolved into four simple stratum submodels, in accordance with the stratification of the experimental units (the sub-plots). Using Nelder's (1965, p. 150) notation, also adopted by Pearce (1983, p. 153), this stratification ("block-structure") can be represented by the relation

$$
\text { Sub-plots } \rightarrow \text { Main plots } \rightarrow \text { Blocks } \rightarrow \text { Total experimental area. }
$$

Thus, the observed vector $\boldsymbol{y}$ can be decomposed as

$$
\boldsymbol{y}=\boldsymbol{y}_{1}+\boldsymbol{y}_{2}+\boldsymbol{y}_{3}+\boldsymbol{y}_{4}, \quad \text { where }
$$




$$
\boldsymbol{y}_{1}=\phi_{1} \boldsymbol{y}, \boldsymbol{y}_{2}=\phi_{2} \boldsymbol{y}, \quad \boldsymbol{y}_{3}=\phi_{3} \boldsymbol{y}, \boldsymbol{y}_{4}=\phi_{4} \boldsymbol{y},
$$

with the applied projectors of the form

$$
\begin{aligned}
& \phi_{1}=\boldsymbol{I}_{n}-k_{2}^{-1} \boldsymbol{X}_{3} \boldsymbol{X}_{3}^{\prime}, \quad \phi_{2}=k_{2}^{-1} \boldsymbol{X}_{3} \boldsymbol{X}_{3}^{\prime}-k^{-1} \boldsymbol{X}_{2} \boldsymbol{X}_{2}^{\prime}, \\
& \phi_{3}=k^{-1} \boldsymbol{X}_{2} \boldsymbol{X}_{2}^{\prime}-n^{-1} \mathbf{1}_{n} \mathbf{1}_{n}^{\prime} \text { and } \phi_{4}=n^{-1} \mathbf{1}_{n} \mathbf{1}_{n}^{\prime},
\end{aligned}
$$

where $k=k_{1} k_{2}$. This notation allows the expectation vector and the covariance (dispersion) matrix of $\boldsymbol{y}$ to be written as

$$
\begin{aligned}
& \mathrm{E}(\boldsymbol{y})=\phi_{1} \boldsymbol{X}_{1} \boldsymbol{\tau}+\phi_{2} \boldsymbol{X}_{1} \boldsymbol{\tau}+\phi_{3} \boldsymbol{X}_{1} \boldsymbol{\tau}+\phi_{4} \boldsymbol{X}_{1} \boldsymbol{\tau}=\boldsymbol{X}_{1} \boldsymbol{\tau}, \\
& \mathrm{D}(\boldsymbol{y}) \equiv \boldsymbol{V}=\sigma_{1}^{2} \phi_{1}+\sigma_{2}^{2} \phi_{2}+\sigma_{3}^{2} \phi_{3}+\sigma_{4}^{2} \phi_{4} .
\end{aligned}
$$

Note that the four projectors used above are symmetric, idempotent and pairwise orthogonal, summing to the identity matrix, and the scalars $\sigma_{1}^{2}$, $\sigma_{2}^{2}, \sigma_{3}^{2}$ and $\sigma_{4}^{2}$ represent the relevant unknown stratum variances (defined similarly as in Caliński and Kageyama, 2000, Section 5.4).

The above results coincide with those presented by I. Mejza (1996, p. 70).

The model (1) analyzed here can easily be modified for a straight extension of the considered simple split-plot design, for example into a splitsplit-plot design.

\section{Theoretical background of the analysis}

When analyzing data from an experiment modeled by (1), a factorial experiment in particular, attention is usually paid to estimates and tests concerning the parameters $\boldsymbol{\tau}=\left[\tau_{1}, \tau_{2}, \ldots, \tau_{v}\right]^{\prime}$, or rather the treatment comparative effects, defined as

$\left(\boldsymbol{I}_{v}-n^{-1} \mathbf{1}_{v} \boldsymbol{r}^{\prime}\right) \boldsymbol{\tau}=\left[\tau_{1}-\tau ., \tau_{2}-\tau ., \ldots, \tau_{v}-\tau .\right]^{\prime}$, where $\tau .=n^{-1} \sum_{i=1}^{v}\left(r_{i} \tau_{i}\right)$,

and also their linear functions (for example the factorial main and interaction effects). In connection with this, first note (referring, for example, to Caliński and Siatkowski, 2018, p. 151) that, taking the orthogonal $\left(\boldsymbol{V}^{-1}\right.$ orthogonal) projector

$$
\boldsymbol{P}_{X_{1}\left(V^{-1}\right)}=\boldsymbol{X}_{1}\left(\boldsymbol{X}_{1}^{\prime} \boldsymbol{V}^{-1} \boldsymbol{X}_{1}\right)^{-1} \boldsymbol{X}_{1}^{\prime} \boldsymbol{V}^{-1},
$$

one can decompose the vector $\boldsymbol{y}$ in (1) into two uncorrelated parts, as

$$
\boldsymbol{y}=\boldsymbol{P}_{X_{1}\left(V^{-1}\right)} \boldsymbol{y}+\left(\boldsymbol{I}_{n}-\boldsymbol{P}_{X_{1}\left(V^{-1}\right)}\right) \boldsymbol{y} .
$$


The role of the two parts on the right in (5) can easily be seen.

Under the model (1), with properties (2) and (3), the first term of the partition in (5) provides the best linear unbiased estimator (BLUE) of $\boldsymbol{X}_{1} \boldsymbol{\tau}$ appearing in (2), which can be expressed as

$$
\widehat{\boldsymbol{X}_{1} \boldsymbol{\tau}}=\boldsymbol{P}_{X_{1}\left(V^{-1}\right)} \boldsymbol{y}
$$

as follows from Rao (1974, Theorem 3.2). With regard to the second part in (5), it can be seen as the residual vector, giving the residual sum of squares, $\mathrm{SS}_{R}$, in the form

$$
\begin{aligned}
\left\|\left(\boldsymbol{I}_{n}-\boldsymbol{P}_{X_{1}\left(V^{-1}\right)}\right) \boldsymbol{y}\right\|_{V^{-1}}^{2} & =\boldsymbol{y}^{\prime}\left(\boldsymbol{I}_{n}-\boldsymbol{P}_{X_{1}\left(V^{-1}\right)}\right)^{\prime} \boldsymbol{V}^{-1}\left(\boldsymbol{I}_{n}-\boldsymbol{P}_{X_{1}\left(V^{-1}\right)}\right) \boldsymbol{y} \\
& =\boldsymbol{y}^{\prime}\left[\boldsymbol{V}^{-1}-\boldsymbol{V}^{-1} \boldsymbol{X}_{1}\left(\boldsymbol{X}_{1}^{\prime} \boldsymbol{V}^{-1} \boldsymbol{X}_{1}\right)^{-1} \boldsymbol{X}_{1}^{\prime} \boldsymbol{V}^{-1}\right] \boldsymbol{y} \\
& =\boldsymbol{y}^{\prime} \boldsymbol{V}^{-1}\left(\boldsymbol{I}_{n}-\boldsymbol{P}_{X_{1}\left(V^{-1}\right)}\right) \boldsymbol{y}
\end{aligned}
$$

with the residual degrees of freedom given by $\operatorname{rank}\left(\boldsymbol{V}: \boldsymbol{X}_{1}\right)-\operatorname{rank}\left(\boldsymbol{X}_{1}\right)=$ $n-v$. See Rao (1974, Theorem 3.4) and formula (3.17) there.

For convenience note that, when using the projector (4) in the considered applications, the variance $\sigma_{4}^{2}$ in the involved matrix $\boldsymbol{V}$, defined in (3), can be replaced by 1 . This is evident from the application of a relevant spectral decomposition, as presented in Caliński and Siatkowski (2018, Section 3).

It will also be interesting to note that, as $\boldsymbol{\tau}=\boldsymbol{r}^{-\delta} \boldsymbol{X}_{1}^{\prime} \boldsymbol{X}_{1} \boldsymbol{\tau}$, the BLUE of $\boldsymbol{\tau}$ can be obtained, by (4) and (6), as

$$
\hat{\boldsymbol{\tau}}=\left(\boldsymbol{X}_{1}^{\prime} \boldsymbol{V}^{-1} \boldsymbol{X}_{1}\right)^{-1} \boldsymbol{X}_{1}^{\prime} \boldsymbol{V}^{-1} \boldsymbol{y} .
$$

Its covariance (dispersion) matrix then takes the form

$$
\begin{aligned}
\mathrm{D}(\hat{\boldsymbol{\tau}}) & =\left(\boldsymbol{X}_{1}^{\prime} \boldsymbol{V}^{-1} \boldsymbol{X}_{1}\right)^{-1} \boldsymbol{X}_{1}^{\prime} \boldsymbol{V}^{-1} \mathrm{D}(\boldsymbol{y}) \boldsymbol{V}^{-1} \boldsymbol{X}_{1}\left(\boldsymbol{X}_{1}^{\prime} \boldsymbol{V}^{-1} \boldsymbol{X}_{1}\right)^{-1} \\
& =\left(\boldsymbol{X}_{1}^{\prime} \boldsymbol{V}^{-1} \boldsymbol{X}_{1}\right)^{-1} .
\end{aligned}
$$

The results (7)-(9) can be checked by referring to Theorem 3.1 in Rao (1971), as shown in Caliński and Siatkowski (2018, p. 152).

With these results the concept of testing the hypothesis

$$
H_{0}:\left(\boldsymbol{I}_{v}-n^{-1} \mathbf{1}_{v} \boldsymbol{r}^{\prime}\right) \boldsymbol{\tau}=\mathbf{0}
$$

can be considered. To see whether the hypothesis (10) is consistent, note that the BLUE of $\boldsymbol{\tau}_{*}=\left(\boldsymbol{I}_{v}-n^{-1} \mathbf{1}_{v} \boldsymbol{r}^{\prime}\right) \boldsymbol{\tau}$ is $\hat{\boldsymbol{\tau}}_{*}=\left(\boldsymbol{I}_{v}-n^{-1} \mathbf{1}_{v} \boldsymbol{r}^{\prime}\right) \hat{\boldsymbol{\tau}}$, with $\hat{\boldsymbol{\tau}}$ as given in (8). Its dispersion matrix is, by (9), of the form

$$
\mathrm{D}\left(\hat{\boldsymbol{\tau}}_{*}\right)=\left(\boldsymbol{I}_{v}-n^{-1} \mathbf{1}_{v} \boldsymbol{r}^{\prime}\right)\left(\boldsymbol{X}_{1}^{\prime} \boldsymbol{V}^{-1} \boldsymbol{X}_{1}\right)^{-1}\left(\boldsymbol{I}_{v}-n^{-1} \boldsymbol{r} \mathbf{1}_{v}^{\prime}\right),
$$


with rank $v-1$. It appears that as a $g$-inverse of $\mathrm{D}\left(\hat{\boldsymbol{\tau}}_{*}\right)$ one can take $\left[\mathrm{D}\left(\hat{\boldsymbol{\tau}}_{*}\right)\right]^{-}=\boldsymbol{X}_{1}^{\prime} \boldsymbol{V}^{-1} \boldsymbol{X}_{1}$. Hence,

$$
\mathrm{D}\left(\hat{\boldsymbol{\tau}}_{*}\right)\left[\mathrm{D}\left(\hat{\boldsymbol{\tau}}_{*}\right)\right]^{-} \hat{\boldsymbol{\tau}}_{*}=\hat{\boldsymbol{\tau}}_{*},
$$

as can be shown (see Appendix 1 in Caliński and Siatkowski, 2018). The equality (12) indicates that $H_{0}$ in (10) is consistent; see formula (3.2.8) in Rao (1971).

Assuming now that $\boldsymbol{y} \sim N_{n}\left(\boldsymbol{X}_{1} \boldsymbol{\tau}, \boldsymbol{V}\right)$ and, hence, that $\hat{\boldsymbol{\tau}}_{*} \sim N_{v}\left[\boldsymbol{\tau}_{*}, \mathrm{D}\left(\hat{\boldsymbol{\tau}}_{*}\right)\right]$, where $\boldsymbol{\tau}_{*}$ is as defined above, and $\mathrm{D}\left(\hat{\boldsymbol{\tau}}_{*}\right)$ is as in (11), one can test the hypothesis $H_{0}$ by the statistic

$$
F=\frac{n-v}{v-1} \frac{\mathrm{SS}_{V}}{\mathrm{SS}_{R}}=\frac{n-v}{v-1} \frac{\hat{\boldsymbol{\tau}}_{*}^{\prime} \boldsymbol{X}_{1}^{\prime} \boldsymbol{V}^{-1} \boldsymbol{X}_{1} \hat{\boldsymbol{\tau}}_{*}}{\boldsymbol{y}^{\prime} \boldsymbol{V}^{-1}\left(\boldsymbol{I}_{n}-\boldsymbol{P}_{X_{1}\left(V^{-1}\right)}\right) \boldsymbol{y}},
$$

as follows from Theorem 3.2 in Rao (1971). Note, however, that the sums of squares in (13) can equivalently be written (see Appendix 2 in Caliński and Siatkowski, 2018) as

$$
\begin{aligned}
& \mathrm{SS}_{V}=\boldsymbol{y}^{\prime} \boldsymbol{V}^{-1} \boldsymbol{X}_{1}\left(\boldsymbol{I}_{v}-\mathbf{1}_{v} \boldsymbol{r}^{\prime} / n\right)\left(\boldsymbol{X}_{1}^{\prime} \boldsymbol{V}^{-1} \boldsymbol{X}_{1}\right)^{-1}\left(\boldsymbol{I}_{v}-\boldsymbol{r} \mathbf{1}_{v}^{\prime} / n\right) \boldsymbol{X}_{1}^{\prime} \boldsymbol{V}^{-1} \boldsymbol{y} \\
& \mathrm{SS}_{R}=\boldsymbol{y}^{\prime}\left[\boldsymbol{V}^{-1}-\boldsymbol{V}^{-1} \boldsymbol{X}_{1}\left(\boldsymbol{X}_{1}^{\prime} \boldsymbol{V}^{-1} \boldsymbol{X}_{1}\right)^{-1} \boldsymbol{X}_{1}^{\prime} \boldsymbol{V}^{-1}\right] \boldsymbol{y}
\end{aligned}
$$

Referring now to Theorems 9.2.1 and 9.4.1 in Rao and Mitra (1971), one can show that, independently,

$$
\begin{aligned}
& \mathrm{SS}_{V} \sim \chi^{2}(v-1, \delta), \quad \text { with } \quad \delta=\boldsymbol{\tau}_{*}^{\prime} \boldsymbol{X}_{1}^{\prime} \boldsymbol{V}^{-1} \boldsymbol{X}_{1} \boldsymbol{\tau}_{*}, \\
& \mathrm{SS}_{R} \sim \chi^{2}(n-v, 0) .
\end{aligned}
$$

Evidently, the distribution in (16) is central if the hypothesis $H_{0}$ is true, whereas that in (17) is central whether $H_{0}$ is true or not. These results imply that the statistic (13) has a noncentral $F$ distribution with $v-1$ and $n-v$ d.f., and with the noncentrality parameter $\delta$ as in (16). Thus, the distribution is central if $H_{0}$ is true.

It should be noted, however, that the above estimation and hypothesis testing procedures are applicable directly if the stratum variances $\sigma_{1}^{2}, \sigma_{2}^{2}$, $\sigma_{3}^{2}$ and $\sigma_{4}^{2}$ are known. In practice they are usually unknown and have to be estimated. To do this, it will be helpful to return to formula (7), writing it as

$$
\left\|\left(\boldsymbol{I}_{n}-\boldsymbol{P}_{X_{1}\left(V^{-1}\right)}\right) \boldsymbol{y}\right\|_{V^{-1}}^{2}
$$




$$
\begin{aligned}
= & \boldsymbol{y}^{\prime}\left(\boldsymbol{I}_{n}-\boldsymbol{P}_{X_{1}\left(V^{-1}\right)}\right)^{\prime} \boldsymbol{V}^{-1}\left(\boldsymbol{I}_{n}-\boldsymbol{P}_{X_{1}\left(V^{-1}\right)}\right) \boldsymbol{y} \\
= & \sigma_{1}^{-2} \boldsymbol{y}^{\prime}\left(\boldsymbol{I}_{n}-\boldsymbol{P}_{X_{1}\left(V^{-1}\right)}\right)^{\prime} \phi_{1}\left(\boldsymbol{I}_{n}-\boldsymbol{P}_{X_{1}\left(V^{-1}\right)}\right) \boldsymbol{y} \\
& +\sigma_{2}^{-2} \boldsymbol{y}^{\prime}\left(\boldsymbol{I}_{n}-\boldsymbol{P}_{X_{1}\left(V^{-1}\right)}\right)^{\prime} \boldsymbol{\phi}_{2}\left(\boldsymbol{I}_{n}-\boldsymbol{P}_{X_{1}\left(V^{-1}\right)}\right) \boldsymbol{y} \\
& +\sigma_{3}^{-2} \boldsymbol{y}^{\prime}\left(\boldsymbol{I}_{n}-\boldsymbol{P}_{X_{1}\left(V^{-1}\right)}\right)^{\prime} \boldsymbol{\phi}_{3}\left(\boldsymbol{I}_{n}-\boldsymbol{P}_{X_{1}\left(V^{-1}\right)}\right) \boldsymbol{y},
\end{aligned}
$$

which follows from the form of $\mathrm{D}(\boldsymbol{y}) \equiv \boldsymbol{V}$, given in (3). This form also implies, on account of the relation $\boldsymbol{\phi}_{4}=n^{-1} \mathbf{1}_{n} \mathbf{1}_{n}^{\prime}=n^{-1} \mathbf{1}_{n} \mathbf{1}_{v}^{\prime} \boldsymbol{X}_{1}^{\prime}$, that

$$
\boldsymbol{\phi}_{4}\left(\boldsymbol{I}_{n}-\boldsymbol{P}_{X_{1}\left(V^{-1}\right)}\right)=\sigma_{4}^{2} \boldsymbol{\phi}_{4} \boldsymbol{V}^{-1}\left(\boldsymbol{I}_{n}-\boldsymbol{P}_{X_{1}\left(V^{-1}\right)}\right)=\mathbf{O} .
$$

It may be interesting to note that one can present the $\mathrm{SS}_{V}$, given in (14), in a form similar to (18), i.e., as

$$
\begin{aligned}
\mathrm{SS}_{V}= & \boldsymbol{y}^{\prime} \boldsymbol{P}_{X_{1}\left(V^{-1}\right)}^{\prime}\left(\boldsymbol{I}_{n}-n^{-1} \mathbf{1}_{n} \mathbf{1}_{n}^{\prime}\right) \boldsymbol{V}^{-1}\left(\boldsymbol{I}_{n}-n^{-1} \mathbf{1}_{n} \mathbf{1}_{n}^{\prime}\right) \boldsymbol{P}_{X_{1}\left(V^{-1}\right)} \boldsymbol{y} \\
= & \sigma_{1}^{-2} \boldsymbol{y}^{\prime} \boldsymbol{P}_{X_{1}\left(V^{-1}\right)}^{\prime} \boldsymbol{\phi}_{1} \boldsymbol{P}_{X_{1}\left(V^{-1}\right)} \boldsymbol{y} \\
& +\sigma_{2}^{-2} \boldsymbol{y}^{\prime} \boldsymbol{P}_{X_{1}\left(V^{-1}\right)}^{\prime} \boldsymbol{\phi}_{2} \boldsymbol{P}_{X_{1}\left(V^{-1}\right)} \boldsymbol{y} \\
& +\sigma_{3}^{-2} \boldsymbol{y}^{\prime} \boldsymbol{P}_{X_{1}\left(V^{-1}\right)}^{\prime} \boldsymbol{\phi}_{3} \boldsymbol{P}_{X_{1}\left(V^{-1}\right)} \boldsymbol{y},
\end{aligned}
$$

which follows from Appendices 1 and 2 in Caliński and Siatkowski (2018).

Now, from (18), one can write

$$
\begin{aligned}
\mathrm{E}\left\{\left\|\left(\boldsymbol{I}_{n}-\boldsymbol{P}_{X_{1}\left(V^{-1}\right)}\right) \boldsymbol{y}\right\|_{V^{-1}}^{2}\right\}= & \sigma_{1}^{-2} \mathrm{E}\left\{\left\|\phi_{1}\left(\boldsymbol{I}_{n}-\boldsymbol{P}_{X_{1}\left(V^{-1}\right)}\right) \boldsymbol{y}\right\|^{2}\right\} \\
& +\sigma_{2}^{-2} \mathrm{E}\left\{\left\|\phi_{2}\left(\boldsymbol{I}_{n}-\boldsymbol{P}_{X_{1}\left(V^{-1}\right)}\right) \boldsymbol{y}\right\|^{2}\right\} \\
& +\sigma_{3}^{-2} \mathrm{E}\left\{\left\|\phi_{3}\left(\boldsymbol{I}_{n}-\boldsymbol{P}_{X_{1}\left(V^{-1}\right)}\right) \boldsymbol{y}\right\|^{2}\right\} \\
= & d_{1}^{\prime}+d_{2}^{\prime}+d_{3}^{\prime}=n-v,
\end{aligned}
$$

because, as can be shown,

$\mathrm{E}\left\{\left\|\boldsymbol{\phi}_{1}\left(\boldsymbol{I}_{n}-\boldsymbol{P}_{X_{1}\left(V^{-1}\right)}\right) \boldsymbol{y}\right\|^{2}\right\}=\sigma_{1}^{2} d_{1}^{\prime}$, where $d_{1}^{\prime}=\operatorname{tr}\left[\boldsymbol{\phi}_{1}\left(\boldsymbol{I}_{n}-\boldsymbol{P}_{X_{1}\left(V^{-1}\right)}\right)\right]$,

$\mathrm{E}\left\{\left\|\boldsymbol{\phi}_{2}\left(\boldsymbol{I}_{n}-\boldsymbol{P}_{X_{1}\left(V^{-1}\right)}\right) \boldsymbol{y}\right\|^{2}\right\}=\sigma_{2}^{2} d_{2}^{\prime}$, where $d_{2}^{\prime}=\operatorname{tr}\left[\boldsymbol{\phi}_{2}\left(\boldsymbol{I}_{n}-\boldsymbol{P}_{X_{1}\left(V^{-1}\right)}\right)\right]$,

$\mathrm{E}\left\{\left\|\phi_{3}\left(\boldsymbol{I}_{n}-\boldsymbol{P}_{X_{1}\left(V^{-1}\right)}\right) \boldsymbol{y}\right\|^{2}\right\}=\sigma_{3}^{2} d_{3}^{\prime}$, where $d_{3}^{\prime}=\operatorname{tr}\left[\boldsymbol{\phi}_{3}\left(\boldsymbol{I}_{n}-\boldsymbol{P}_{X_{1}\left(V^{-1}\right)}\right)\right]$

With these results it is natural to use as estimators of $\sigma_{1}^{2}, \sigma_{2}^{2}$ and $\sigma_{3}^{2}$ the solutions of the equations

$$
\begin{aligned}
\left\|\phi_{1}\left(\boldsymbol{I}_{n}-\boldsymbol{P}_{X_{1}\left(V^{-1}\right)}\right) \boldsymbol{y}\right\|^{2} & =\sigma_{1}^{2} d_{1}^{\prime}, \\
\left\|\phi_{2}\left(\boldsymbol{I}_{n}-\boldsymbol{P}_{X_{1}\left(V^{-1}\right)}\right) \boldsymbol{y}\right\|^{2} & =\sigma_{2}^{2} d_{2}^{\prime}, \\
\left\|\boldsymbol{\phi}_{3}\left(\boldsymbol{I}_{n}-\boldsymbol{P}_{X_{1}\left(V^{-1}\right)}\right) \boldsymbol{y}\right\|^{2} & =\sigma_{3}^{2} d_{3}^{\prime},
\end{aligned}
$$


respectively (as suggested by Nelder, 1968, Section 3). This approach was also advocated by Houtman and Speed (1983, Section 4.5) and is often applied in practice.

For completeness, it will be helpful to note that the equations (23), (24) and (25), with the formulae (20), (21) and (22), imply - on account of (19) - the equality

$$
\begin{aligned}
\hat{\sigma}_{1}^{-2}\left\|\boldsymbol{\phi}_{1}\left(\boldsymbol{I}_{n}-\boldsymbol{P}_{X_{1}\left(V^{-1}\right)}\right) \boldsymbol{y}\right\|^{2}+ & \hat{\sigma}_{2}^{-2}\left\|\boldsymbol{\phi}_{2}\left(\boldsymbol{I}_{n}-\boldsymbol{P}_{X_{1}\left(V^{-1}\right)}\right) \boldsymbol{y}\right\|^{2} \\
+ & \hat{\sigma}_{3}^{-2}\left\|\boldsymbol{\phi}_{3}\left(\boldsymbol{I}_{n}-\boldsymbol{P}_{X_{1}\left(V^{-1}\right)}\right) \boldsymbol{y}\right\|^{2} \\
& =d_{1}^{\prime}+d_{2}^{\prime}+d_{3}^{\prime}=n-v .
\end{aligned}
$$

Now, proceeding as in Caliński and Siatkowski (2018, p. 155), it can be shown that

$$
\begin{aligned}
\mathrm{SS}_{R}= & \sigma_{1}^{-2}\left\|\boldsymbol{\phi}_{1}\left(\boldsymbol{I}_{n}-\boldsymbol{P}_{X_{1}\left(V^{-1}\right)}\right) \boldsymbol{y}\right\|^{2}+\sigma_{2}^{-2}\left\|\boldsymbol{\phi}_{2}\left(\boldsymbol{I}_{n}-\boldsymbol{P}_{X_{1}\left(V^{-1}\right)}\right) \boldsymbol{y}\right\|^{2} \\
& +\sigma_{3}^{-2}\left\|\boldsymbol{\phi}_{3}\left(\boldsymbol{I}_{n}-\boldsymbol{P}_{X_{1}\left(V^{-1}\right)}\right) \boldsymbol{y}\right\|^{2}
\end{aligned}
$$

A comparison of formulae (26) and (27) shows that if the stratum variances are estimated, by solutions of the equations (23), (24) and (25), then

$$
\begin{aligned}
\widehat{\mathrm{SS}}_{R}= & \hat{\sigma}_{1}^{-2}\left\|\boldsymbol{\phi}_{1}\left(\boldsymbol{I}_{n}-\boldsymbol{P}_{X_{1}\left(V^{-1}\right)}\right) \boldsymbol{y}\right\|^{2}+\hat{\sigma}_{2}^{-2}\left\|\boldsymbol{\phi}_{2}\left(\boldsymbol{I}_{n}-\boldsymbol{P}_{X_{1}\left(V^{-1}\right)}\right) \boldsymbol{y}\right\|^{2} \\
& +\hat{\sigma}_{3}^{-2}\left\|\boldsymbol{\phi}_{3}\left(\boldsymbol{I}_{n}-\boldsymbol{P}_{X_{1}\left(V^{-1}\right)}\right) \boldsymbol{y}\right\|^{2}=n-v
\end{aligned}
$$

By (28), the statistic $F$ in (13) is reduced to the form

$$
\widehat{F}=\frac{n-v}{v-1} \frac{\widehat{\mathrm{SS}}_{V}}{n-v}=\frac{\widehat{\mathrm{SS}}_{V}}{v-1}
$$

where $\widehat{\mathrm{SS}}_{V}$ is as in (14), but with $\sigma_{1}^{2}, \sigma_{2}^{2}$ and $\sigma_{3}^{2}$ there replaced by their estimates.

However, the $\chi^{2}$ distribution of $\mathrm{SS}_{V}$, indicated in (16), is valid exactly only if the true stratum variances are used in the applied matrix $\boldsymbol{V}^{-1}=$ $\sigma_{1}^{-2} \boldsymbol{\phi}_{1}+\sigma_{2}^{-2} \boldsymbol{\phi}_{2}+\sigma_{3}^{-2} \boldsymbol{\phi}_{3}+\sigma_{4}^{-2} \boldsymbol{\phi}_{4}$, resulting from (3). As for the component $\sigma_{4}^{-2} \phi_{4}$, it does not in fact play any role in the application of formula (14) given for $\mathrm{SS}_{V}$ (as will be shown in the next section). Thus, when using in $\boldsymbol{V}^{-1}$ the estimates of $\sigma_{1}^{2}, \sigma_{2}^{2}$ and $\sigma_{3}^{2}$ obtained from (23), (24) and (25) respectively, the distribution (16) can be regarded as approximate only. 


\section{Some simplifying reformulations}

As noted in the previous section, the component $\sigma_{4}^{-2} \boldsymbol{\phi}_{4}$ in $\boldsymbol{V}^{-1}=\sigma_{1}^{-2} \boldsymbol{\phi}_{1}+$ $\sigma_{2}^{-2} \phi_{2}+\sigma_{3}^{-2} \phi_{3}+\sigma_{4}^{-2} \phi_{4}$ seems to play no role in the formulae applicable in the considered analysis of experimental data. Hence, some reformulation in the methodology presented in Section 3 would simplify the analysis, without causing any changes in its results.

A desirable simplification can be obtained when the dispersion matrix $\boldsymbol{V}$ given in (3) is replaced by the matrix $\boldsymbol{V}_{*}=\sigma_{1}^{2} \phi_{1}+\sigma_{2}^{2} \phi_{2}+\sigma_{3}^{2}\left(\phi_{3}+\phi_{4}\right)$, i.e., when the inverted matrix $\boldsymbol{V}^{-1}$ is replaced by

$$
\boldsymbol{V}_{*}^{-1}=\sigma_{1}^{-2} \phi_{1}+\sigma_{2}^{-2} \phi_{2}+\sigma_{3}^{-2}\left(\phi_{3}+\phi_{4}\right) .
$$

The relations between $\boldsymbol{V}$ and $\boldsymbol{V}_{*}$, and their inverses, are given by

$$
\begin{aligned}
& \boldsymbol{V}=\boldsymbol{V}_{*}+\left(\sigma_{4}^{2}-\sigma_{3}^{2}\right) n^{-1} \mathbf{1}_{n} \mathbf{1}_{n}^{\prime} \text { and } \\
& \boldsymbol{V}^{-1}=\boldsymbol{V}_{*}^{-1}+\left(\sigma_{4}^{-2}-\sigma_{3}^{-2}\right) n^{-1} \mathbf{1}_{n} \mathbf{1}_{n}^{\prime} .
\end{aligned}
$$

From (30) it follows (see Appendix 3 in Caliński and Siatkowski, 2018) that

$$
\left(\boldsymbol{X}_{1}^{\prime} \boldsymbol{V}^{-1} \boldsymbol{X}_{1}\right)^{-1}=\left(\boldsymbol{X}_{1}^{\prime} \boldsymbol{V}_{*}^{-1} \boldsymbol{X}_{1}\right)^{-1}+\left(\sigma_{4}^{2}-\sigma_{3}^{2}\right) n^{-1} \mathbf{1}_{v} \mathbf{1}_{v}^{\prime},
$$

and that the BLUE of $\boldsymbol{\tau}_{*}=\left(\boldsymbol{I}_{v}-n^{-1} \mathbf{1}_{v} \boldsymbol{r}^{\prime}\right) \boldsymbol{\tau}$ following from (8), i.e.,

$$
\hat{\boldsymbol{\tau}}_{*}=\left(\boldsymbol{I}_{v}-n^{-1} \mathbf{1}_{v} \boldsymbol{r}^{\prime}\right) \hat{\boldsymbol{\tau}}=\left(\boldsymbol{I}_{v}-n^{-1} \mathbf{1}_{v} \boldsymbol{r}^{\prime}\right)\left(\boldsymbol{X}_{1}^{\prime} \boldsymbol{V}^{-1} \boldsymbol{X}_{1}\right)^{-1} \boldsymbol{X}_{1}^{\prime} \boldsymbol{V}^{-1} \boldsymbol{y},
$$

can equivalently be written as

$$
\hat{\boldsymbol{\tau}}_{*}=\left(\boldsymbol{I}_{v}-n^{-1} \mathbf{1}_{v} \boldsymbol{r}^{\prime}\right)\left(\boldsymbol{X}_{1}^{\prime} \boldsymbol{V}_{*}^{-1} \boldsymbol{X}_{1}\right)^{-1} \boldsymbol{X}_{1}^{\prime} \boldsymbol{V}_{*}^{-1} \boldsymbol{y}_{*},
$$

where $\boldsymbol{y}_{*}=\left(\boldsymbol{I}_{n}-n^{-1} \mathbf{1}_{n} \mathbf{1}_{n}^{\prime}\right) \boldsymbol{y}$, for which

$$
\mathrm{E}\left(\boldsymbol{y}_{*}\right)=\left(\boldsymbol{I}_{n}-n^{-1} \mathbf{1}_{n} \mathbf{1}_{n}^{\prime}\right) \boldsymbol{X}_{1} \boldsymbol{\tau}=\boldsymbol{X}_{1}\left(\boldsymbol{I}_{v}-n^{-1} \mathbf{1}_{v} \boldsymbol{r}^{\prime}\right) \boldsymbol{\tau}=\boldsymbol{X}_{1} \boldsymbol{\tau}_{*}
$$

and

$$
\mathrm{D}\left(\boldsymbol{y}_{*}\right)=\left(\boldsymbol{I}_{n}-n^{-1} \mathbf{1}_{n} \mathbf{1}_{n}^{\prime}\right) \boldsymbol{V}_{*}\left(\boldsymbol{I}_{n}-n^{-1} \mathbf{1}_{n} \mathbf{1}_{n}^{\prime}\right) .
$$

The dispersion matrix of $\hat{\boldsymbol{\tau}}_{*}$, given in (11), can on account of (31) be presented as

$$
\mathrm{D}\left(\hat{\boldsymbol{\tau}}_{*}\right)=\left(\boldsymbol{I}_{v}-n^{-1} \mathbf{1}_{v} \boldsymbol{r}^{\prime}\right)\left(\boldsymbol{X}_{1}^{\prime} \boldsymbol{V}_{*}^{-1} \boldsymbol{X}_{1}\right)^{-1}\left(\boldsymbol{I}_{v}-n^{-1} \boldsymbol{r} \mathbf{1}_{v}^{\prime}\right) .
$$


Furthermore, proceeding as in Caliński and Siatkowski (2018, Appendices 3 and 4), one can write

$$
\begin{aligned}
& \mathrm{SS}_{V}=\hat{\boldsymbol{\tau}}_{*}^{\prime} \boldsymbol{X}_{1}^{\prime} \boldsymbol{V}_{*}^{-1} \boldsymbol{X}_{1} \hat{\boldsymbol{\tau}}_{*}=\boldsymbol{y}_{*}^{\prime} \boldsymbol{V}_{*}^{-1} \boldsymbol{X}_{1}\left(\boldsymbol{X}_{1}^{\prime} \boldsymbol{V}_{*}^{-1} \boldsymbol{X}_{1}\right)^{-1} \boldsymbol{X}_{1}^{\prime} \boldsymbol{V}_{*}^{-1} \boldsymbol{y}_{*}, \\
& \mathrm{SS}_{R}=\boldsymbol{y}_{*}^{\prime}\left[\boldsymbol{V}_{*}^{-1}-\boldsymbol{V}_{*}^{-1} \boldsymbol{X}_{1}\left(\boldsymbol{X}_{1}^{\prime} \boldsymbol{V}_{*}^{-1} \boldsymbol{X}_{1}\right)^{-1} \boldsymbol{X}_{1}^{\prime} \boldsymbol{V}_{*}^{-1}\right] \boldsymbol{y}_{*},
\end{aligned}
$$

with $\boldsymbol{y}_{*}=\left(\boldsymbol{I}_{n}-n^{-1} \mathbf{1}_{n} \mathbf{1}_{n}^{\prime}\right) \boldsymbol{y}$. The formulae (34) and (35) provide the sum

$$
\mathrm{SS}_{V}+\mathrm{SS}_{R}=\boldsymbol{y}_{*}^{\prime} \boldsymbol{V}_{*}^{-1} \boldsymbol{y}_{*}=\mathrm{SS}_{T} \quad \text { (say), }
$$

which can be called the total sum of squares. Referring again to Rao and Mitra (1971, Theorem 9.2.1), it can be shown that

$$
\mathrm{SS}_{T} \sim \chi^{2}(n-1, \delta), \quad \text { with } \delta=\boldsymbol{\tau}_{*}^{\prime} \boldsymbol{X}_{1}^{\prime} \boldsymbol{V}_{*}^{-1} \boldsymbol{X}_{1} \boldsymbol{\tau}_{*}
$$

equivalent to $\delta$ as given in (16). These results can be summarized in the form of an ANOVA table, as presented in Table 1.

Table 1. Analysis of variance for an experiment in a split-plot design with orthogonal block structure

\begin{tabular}{lccc}
\hline $\begin{array}{c}\text { Source } \\
\text { of variation }\end{array}$ & $\begin{array}{c}\text { Degrees } \\
\text { of freedom }\end{array}$ & $\begin{array}{c}\text { Sum } \\
\text { of squares }\end{array}$ & $\begin{array}{c}\text { Expected } \\
\text { mean square }\end{array}$ \\
\hline $\begin{array}{l}\text { Treatments } \\
\text { Residuals }\end{array}$ & $v-1$ & $\mathrm{SS}_{V}$ & $1+\delta /(v-1)$ \\
\hline Total & $n-v$ & $\mathrm{SS}_{R}$ & 1 \\
\hline
\end{tabular}

Suppose now that after rejecting the hypothesis (10) one is interested in testing the hypothesis $H_{0, \mathrm{~L}}: \boldsymbol{U}_{\mathrm{L}}^{\prime} \boldsymbol{\tau}=\mathbf{0}$, where $\boldsymbol{U}_{\mathrm{L}}^{\prime} \mathbf{1}_{v}=\mathbf{0}$. Note that this hypothesis, concerning a set of contrasts among treatment parameters, can also be written as

$$
H_{0, \mathrm{~L}}: \boldsymbol{U}_{\mathrm{L}}^{\prime} \boldsymbol{\tau}_{*}=\mathbf{0}, \quad \text { where } \quad \boldsymbol{\tau}_{*}=\left(\boldsymbol{I}_{v}-n^{-1} \mathbf{1}_{v} \boldsymbol{r}^{\prime}\right) \boldsymbol{\tau} .
$$

This shows that $H_{0, \mathrm{~L}}$ is implied by $H_{0}$, given in (10). To find the relevant sum of squares, first note that the BLUE of $\boldsymbol{U}_{\mathrm{L}}^{\prime} \boldsymbol{\tau}_{*}$ is, on account of (32), of the form

$$
\boldsymbol{U}_{\mathrm{L}}^{\prime} \hat{\boldsymbol{\tau}}_{*}=\boldsymbol{U}_{\mathrm{L}}^{\prime} \hat{\boldsymbol{\tau}}=\boldsymbol{U}_{\mathrm{L}}^{\prime}\left(\boldsymbol{X}_{1}^{\prime} \boldsymbol{V}_{*}^{-1} \boldsymbol{X}_{1}\right)^{-1} \boldsymbol{X}_{1}^{\prime} \boldsymbol{V}_{*}^{-1} \boldsymbol{y}_{*} .
$$

Its dispersion matrix is, on account of (33), of the form

$$
\mathrm{D}\left(\boldsymbol{U}_{\mathrm{L}}^{\prime} \hat{\boldsymbol{\tau}}_{*}\right)=\boldsymbol{U}_{\mathrm{L}}^{\prime}\left(\boldsymbol{X}_{1}^{\prime} \boldsymbol{V}_{*}^{-1} \boldsymbol{X}_{1}\right)^{-1} \boldsymbol{U}_{\mathrm{L}}
$$


Note that $\boldsymbol{U}_{\mathrm{L}}^{\prime}\left(\boldsymbol{X}_{1}^{\prime} \boldsymbol{V}_{*}^{-1} \boldsymbol{X}_{1}\right)^{-1} \boldsymbol{U}_{\mathrm{L}}\left[\boldsymbol{U}_{\mathrm{L}}^{\prime}\left(\boldsymbol{X}_{1}^{\prime} \boldsymbol{V}_{*}^{-1} \boldsymbol{X}_{1}\right)^{-1} \boldsymbol{U}_{\mathrm{L}}\right]^{-} \boldsymbol{U}_{\mathrm{L}}^{\prime}=\boldsymbol{U}_{\mathrm{L}}^{\prime}$, which, with (39), gives the equality

$$
\mathrm{D}\left(\boldsymbol{U}_{\mathrm{L}}^{\prime} \hat{\boldsymbol{\tau}}_{*}\right)\left[\mathrm{D}\left(\boldsymbol{U}_{\mathrm{L}}^{\prime} \hat{\boldsymbol{\tau}}_{*}\right)\right]^{-} \boldsymbol{U}_{\mathrm{L}}^{\prime} \hat{\boldsymbol{\tau}}_{*}=\boldsymbol{U}_{\mathrm{L}}^{\prime} \hat{\boldsymbol{\tau}}_{*}
$$

This shows that the hypothesis in (37) is consistent. The relevant sum of squares can then be obtained in the form

$$
\begin{aligned}
\operatorname{SS}\left(\boldsymbol{U}_{\mathrm{L}}\right) & =\hat{\boldsymbol{\tau}}_{*}^{\prime} \boldsymbol{U}_{\mathrm{L}}\left[\mathrm{D}\left(\boldsymbol{U}_{\mathrm{L}}^{\prime} \hat{\boldsymbol{\tau}}_{*}\right)\right]^{-} \boldsymbol{U}_{\mathrm{L}}^{\prime} \hat{\boldsymbol{\tau}}_{*} \\
& =\hat{\boldsymbol{\tau}}_{*}^{\prime} \boldsymbol{U}_{\mathrm{L}}\left[\boldsymbol{U}_{\mathrm{L}}^{\prime}\left(\boldsymbol{X}_{1}^{\prime} \boldsymbol{V}_{*}^{-1} \boldsymbol{X}_{1}\right)^{-1} \boldsymbol{U}_{\mathrm{L}}\right]^{-} \boldsymbol{U}_{\mathrm{L}}^{\prime} \hat{\boldsymbol{\tau}}_{*},
\end{aligned}
$$

with the d.f. equal to $\operatorname{rank}\left(\boldsymbol{U}_{\mathrm{L}}\right)$, i.e., equal to $\operatorname{rank}\left[\mathrm{D}\left(\boldsymbol{U}_{\mathrm{L}}^{\prime} \hat{\boldsymbol{\tau}}_{*}\right)\right]$. Note, referring to Lemma 2.2.6(d) in Rao and Mitra (1971), that

$$
\boldsymbol{U}_{\mathrm{L}}\left[\boldsymbol{U}_{\mathrm{L}}^{\prime}\left(\boldsymbol{X}_{1}^{\prime} \boldsymbol{V}_{*}^{-1} \boldsymbol{X}_{1}\right)^{-1} \boldsymbol{U}_{\mathrm{L}}\right]^{-} \boldsymbol{U}_{\mathrm{L}}^{\prime}
$$

is invariant for any choice of the appearing $g$-inverse, and is of rank equal to the rank of $\boldsymbol{U}_{\mathrm{L}}$. Of course, if the columns of $\boldsymbol{U}_{\mathrm{L}}$ are linearly independent, then

$$
\left[\boldsymbol{U}_{\mathrm{L}}^{\prime}\left(\boldsymbol{X}_{1}^{\prime} \boldsymbol{V}_{*}^{-1} \boldsymbol{X}_{1}\right)^{-1} \boldsymbol{U}_{\mathrm{L}}\right]^{-} \text {becomes }\left[\boldsymbol{U}_{\mathrm{L}}^{\prime}\left(\boldsymbol{X}_{1}^{\prime} \boldsymbol{V}_{*}^{-1} \boldsymbol{X}_{1}\right)^{-1} \boldsymbol{U}_{\mathrm{L}}\right]^{-1} .
$$

Now, following the assumption $\boldsymbol{y} \sim N_{n}\left(\boldsymbol{X}_{1} \boldsymbol{\tau}, \boldsymbol{V}\right)$, one may also assume that $\boldsymbol{U}_{\mathrm{L}}^{\prime} \hat{\boldsymbol{\tau}}_{*} \sim N\left[\boldsymbol{U}_{\mathrm{L}}^{\prime} \boldsymbol{\tau}_{*}, \mathrm{D}\left(\boldsymbol{U}_{\mathrm{L}}^{\prime} \hat{\boldsymbol{\tau}}_{*}\right)\right]$. With this, applying Theorem 9.2.3 from Rao and Mitra (1971), it can be shown that

$$
\operatorname{SS}\left(\boldsymbol{U}_{\mathrm{L}}\right) \sim \chi^{2}\left[\operatorname{rank}\left(\boldsymbol{U}_{\mathrm{L}}\right), \delta_{L}\right], \quad \text { with } \quad \delta_{\mathrm{L}}=\boldsymbol{\tau}_{*}^{\prime} \boldsymbol{U}_{\mathrm{L}}\left[\mathrm{D}\left(\boldsymbol{U}_{\mathrm{L}}^{\prime} \hat{\boldsymbol{\tau}}_{*}\right)\right]^{-} \boldsymbol{U}_{\mathrm{L}}^{\prime} \boldsymbol{\tau}_{*},
$$

this distribution being central, i.e., with $\delta_{\mathrm{L}}=0$, if $H_{0, \mathrm{~L}}$ is true.

If there are several sets of contrasts for which individual hypothesis testing is of interest, then for each of them the sum of squares presented in (40) can be used accordingly. In some situations a relevant partition of the treatment sum of squares, given in (34), may be of interest. The question then arises of what kind of conditions have to be satisfied. It can be shown (see Appendix 5 in Caliński and Siatkowski, 2018) that for two such sets of contrasts, e.g. $\boldsymbol{U}_{\mathrm{A}}^{\prime} \boldsymbol{\tau}_{*}$ and $\boldsymbol{U}_{\mathrm{B}}^{\prime} \boldsymbol{\tau}_{*}$, the equality

$$
\mathrm{SS}\left(\boldsymbol{U}_{\mathrm{A}}\right)+\mathrm{SS}\left(\boldsymbol{U}_{\mathrm{B}}\right)=\mathrm{SS}_{V}
$$

holds, for any vector $\hat{\boldsymbol{\tau}}_{*}=\left(\boldsymbol{I}_{v}-n^{-1} \mathbf{1}_{v} \boldsymbol{r}^{\prime}\right) \hat{\boldsymbol{\tau}}$, if and only if

$$
\begin{aligned}
& \left(\boldsymbol{X}_{1}^{\prime} \boldsymbol{V}_{*}^{-1} \boldsymbol{X}_{1}\right)^{-1} \boldsymbol{U}_{\mathrm{A}}\left[\boldsymbol{U}_{\mathrm{A}}^{\prime}\left(\boldsymbol{X}_{1}^{\prime} \boldsymbol{V}_{*}^{-1} \boldsymbol{X}_{1}\right)^{-1} \boldsymbol{U}_{\mathrm{A}}\right]^{-} \boldsymbol{U}_{\mathrm{A}}^{\prime} \\
+ & \left(\boldsymbol{X}_{1}^{\prime} \boldsymbol{V}_{*}^{-1} \boldsymbol{X}_{1}\right)^{-1} \boldsymbol{U}_{\mathrm{B}}\left[\boldsymbol{U}_{\mathrm{B}}^{\prime}\left(\boldsymbol{X}_{1}^{\prime} \boldsymbol{V}_{*}^{-1} \boldsymbol{X}_{1}\right)^{-1} \boldsymbol{U}_{\mathrm{B}}\right]^{-} \boldsymbol{U}_{\mathrm{B}}^{\prime}=\boldsymbol{I}_{v}-n^{-1} \mathbf{1}_{v} \boldsymbol{r}^{\prime} .
\end{aligned}
$$


This, in turn, implies (on account of Lemma 2.2.6 in Rao and Mitra, 1971) that

$$
\boldsymbol{U}_{\mathrm{B}}^{\prime}\left(\boldsymbol{X}_{1}^{\prime} \boldsymbol{V}_{*}^{-1} \boldsymbol{X}_{1}\right)^{-1} \boldsymbol{U}_{\mathrm{A}}=\mathbf{O} .
$$

These results can be extended for any number of considered sets of contrasts used in a partition of the type (41). The condition (43) can then be written as

$$
\boldsymbol{U}_{\mathrm{L}}^{\prime}\left(\boldsymbol{X}_{1}^{\prime} \boldsymbol{V}_{*}^{-1} \boldsymbol{X}_{1}\right)^{-1} \boldsymbol{U}_{\mathrm{L}^{*}}=\mathbf{O} \quad \text { for } \quad \mathrm{L} \neq \mathrm{L}^{*} .
$$

These comments may be interesting particularly for the analysis of a factorial experiment.

\section{Application with estimated stratum variances}

The hypothesis testing procedures presented in Section 4 are applicable with fully correct distributions if the stratum variances $\sigma_{1}^{2}, \sigma_{2}^{2}$ and $\sigma_{3}^{2}$ are known. As mentioned in Section 3, these variances are usually unknown and have to be estimated. This can be done by solving the equations (23), (24) and (25). However, with these estimates the residual sum of squares $\mathrm{SS}_{R}$ is reduced to $n-v$, the corresponding d.f., as shown in formula (28). This leads to a corresponding reduction of the $F$ statistic (13) to that presented in (29). The estimated treatment sum of squares appearing there, $\widehat{\mathrm{SS}}_{V}$, can, on account of formulae (34), (35) and (36), be written as

$$
\widehat{\mathrm{SS}}_{V}=\boldsymbol{y}_{*}^{\prime} \hat{\boldsymbol{V}}_{*}^{-1} \boldsymbol{y}_{*}-(n-v) \equiv \widehat{\mathrm{SS}}_{T}-n+v .
$$

In the case of known values of $\sigma_{1}^{2}, \sigma_{2}^{2}$ and $\sigma_{3}^{2}$, the total sum of squares $\mathrm{SS}_{T}=\boldsymbol{y}_{*}^{\prime} \boldsymbol{V}_{*}^{-1} \boldsymbol{y}_{*}$ is distributed as $\chi^{2}(n-1, \delta)$. If the hypothesis $H_{0}$ given in (10) is true, then $\delta=0$ and the distribution is central. However, the indicated distribution of $\mathrm{SS}_{T}$ is fully applicable only if the true stratum variances appearing in $\boldsymbol{V}_{*}^{-1}=\sigma_{1}^{-2} \boldsymbol{\phi}_{1}+\sigma_{2}^{-2} \phi_{2}+\sigma_{3}^{-2}\left(\phi_{3}+\phi_{4}\right)$ are used. Because now the matrix $\boldsymbol{V}_{*}^{-1}$ is replaced by

$$
\hat{\boldsymbol{V}}_{*}^{-1}=\hat{\sigma}_{1}^{-2} \boldsymbol{\phi}_{1}+\hat{\sigma}_{2}^{-2} \boldsymbol{\phi}_{2}+\hat{\sigma}_{3}^{-2}\left(\phi_{3}+\phi_{4}\right)
$$

the estimated total sum of squares $\widehat{\mathrm{SS}}_{T}$, appearing in (45), does not have an exact $\chi^{2}$ distribution with $n-1$ d.f. That distribution can, however, be considered as an approximation of the real distribution of $\widehat{\mathrm{SS}}_{T}$. This 
approximation will be the closer the larger is the number $n$, i.e., the size of the experiment.

With this approximation, the estimated mean square $\widehat{\mathrm{MS}}_{V}=\widehat{\mathrm{SS}}_{V} /(v-1)$, denoted by $\widehat{F}$ in (29), may be treated in a practical application as having (under $\left.H_{0}\right)$ approximately the distribution of $\chi^{2}(v-1,0) /(v-1)$, as follows from the relation in (45).

Thus, referring the test statistic (29) to the $\chi^{2}(v-1,0) /(v-1)$ distribution, one will obtain an approximate test of the hypothesis $H_{0}$ formulated in (10). This means that when calculating the relevant $P$ values (i.e., the critical levels of significance) for testing $H_{0}$, or hypotheses implied by $H_{0}$, one has to consider them as approximate. The results obtained by Volaufova (2009) seem to suggest that the above ANOVA type $F$ test approximation will in most cases provide reasonably accurate $P$ values.

Finally, it may be interesting to recall the comments in Johnson, Kotz and Balakrishnan (1995, p. 338) according to which, if in the $F$ statistic as in (13) the d.f. $n-v$ is large, then the natural approximation to be used is that this $F$ statistic is distributed as $\chi^{2}(v-1,0) /(v-1)$. In fact, according to these comments the distribution of the statistic (29) corresponds to the $F$ distribution with the second d.f. tending to infinity; see formula (27.27) there.

\section{Examples}

The analytical methods considered in the previous sections will now be illustrated using data from two experiments conducted in different split-plot designs inducing the OBS property. The first one was conducted in a complete design, and the second in an incomplete split-plot design. As a third example one may consider Example 1 in Caliński and Siatkowski (2018), in which the analyzed experiment was in fact conducted in a split-plot design. All required computations were performed using R (R Core Team, 2017).

Example 1. Elandt (1964, Chapter 8) analyzed data (Table 2) from a $v_{\mathrm{A}} \times$ $v_{\mathrm{B}}$ factorial experiment with $v_{\mathrm{A}}=3$ varieties of barley (factor A) and $v_{\mathrm{B}}=5$ nitrogen fertilization systems (factor B) in $b=6$ blocks. The experiment was conducted in a split-plot design with $\mathcal{D}^{*}$ based on the incidence matrix 


$$
\boldsymbol{N}=\left[\begin{array}{llllllllllllllllll}
1 & 0 & 0 & 1 & 0 & 0 & 1 & 0 & 0 & 1 & 0 & 0 & 1 & 0 & 0 & 1 & 0 & 0 \\
1 & 0 & 0 & 1 & 0 & 0 & 1 & 0 & 0 & 1 & 0 & 0 & 1 & 0 & 0 & 1 & 0 & 0 \\
1 & 0 & 0 & 1 & 0 & 0 & 1 & 0 & 0 & 1 & 0 & 0 & 1 & 0 & 0 & 1 & 0 & 0 \\
1 & 0 & 0 & 1 & 0 & 0 & 1 & 0 & 0 & 1 & 0 & 0 & 1 & 0 & 0 & 1 & 0 & 0 \\
1 & 0 & 0 & 1 & 0 & 0 & 1 & 0 & 0 & 1 & 0 & 0 & 1 & 0 & 0 & 1 & 0 & 0 \\
0 & 1 & 0 & 0 & 1 & 0 & 0 & 1 & 0 & 0 & 1 & 0 & 0 & 1 & 0 & 0 & 1 & 0 \\
0 & 1 & 0 & 0 & 1 & 0 & 0 & 1 & 0 & 0 & 1 & 0 & 0 & 1 & 0 & 0 & 1 & 0 \\
0 & 1 & 0 & 0 & 1 & 0 & 0 & 1 & 0 & 0 & 1 & 0 & 0 & 1 & 0 & 0 & 1 & 0 \\
0 & 1 & 0 & 0 & 1 & 0 & 0 & 1 & 0 & 0 & 1 & 0 & 0 & 1 & 0 & 0 & 1 & 0 \\
0 & 1 & 0 & 0 & 1 & 0 & 0 & 1 & 0 & 0 & 1 & 0 & 0 & 1 & 0 & 0 & 1 & 0 \\
0 & 0 & 1 & 0 & 0 & 1 & 0 & 0 & 1 & 0 & 0 & 1 & 0 & 0 & 1 & 0 & 0 & 1 \\
0 & 0 & 1 & 0 & 0 & 1 & 0 & 0 & 1 & 0 & 0 & 1 & 0 & 0 & 1 & 0 & 0 & 1 \\
0 & 0 & 1 & 0 & 0 & 1 & 0 & 0 & 1 & 0 & 0 & 1 & 0 & 0 & 1 & 0 & 0 & 1 \\
0 & 0 & 1 & 0 & 0 & 1 & 0 & 0 & 1 & 0 & 0 & 1 & 0 & 0 & 1 & 0 & 0 & 1 \\
0 & 0 & 1 & 0 & 0 & 1 & 0 & 0 & 1 & 0 & 0 & 1 & 0 & 0 & 1 & 0 & 0 & 1
\end{array}\right] .
$$

The above partition of the matrix $\boldsymbol{N}$ into the matrices $\boldsymbol{N}_{h}, h=1,2, \ldots, 6$, each composed of three columns, provides the design $\mathcal{D}$ described by the incidence matrix

$$
\boldsymbol{M}=\left[\begin{array}{llllll}
1 & 1 & 1 & 1 & 1 & 1 \\
1 & 1 & 1 & 1 & 1 & 1 \\
1 & 1 & 1 & 1 & 1 & 1 \\
1 & 1 & 1 & 1 & 1 & 1 \\
1 & 1 & 1 & 1 & 1 & 1 \\
1 & 1 & 1 & 1 & 1 & 1 \\
1 & 1 & 1 & 1 & 1 & 1 \\
1 & 1 & 1 & 1 & 1 & 1 \\
1 & 1 & 1 & 1 & 1 & 1 \\
1 & 1 & 1 & 1 & 1 & 1 \\
1 & 1 & 1 & 1 & 1 & 1 \\
1 & 1 & 1 & 1 & 1 & 1 \\
1 & 1 & 1 & 1 & 1 & 1 \\
1 & 1 & 1 & 1 & 1 & 1 \\
1 & 1 & 1 & 1 & 1 & 1
\end{array}\right]
$$


Table 2. Experimental observations of the field plot yield of the combinations of the levels of the two factors analyzed in Example 1

\begin{tabular}{|c|c|c|c|c|c|c|c|c|c|c|c|}
\hline Block & A I & B & Observ. & Block & $\mathrm{A}$ & B & Observ. & Block & & $\mathrm{B}$ & Dbserv. \\
\hline 1 & 1 & & 5.6 & 3 & 1 & 1 & 4.7 & 5 & 1 & 1 & 5.3 \\
\hline 1 & 1 & 2 & 6.4 & 3 & 1 & 2 & 5.2 & 5 & 1 & 2 & 5.2 \\
\hline 1 & 1 & 3 & 7.4 & 3 & 1 & 3 & 6.4 & 5 & 1 & 3 & 6.5 \\
\hline 1 & 1 & 4 & 8.3 & 3 & 1 & 4 & 7.6 & 5 & 1 & 4 & 7.9 \\
\hline 1 & 1 & 5 & 8.2 & 3 & 1 & 5 & 8.2 & 5 & 1 & 5 & 8.4 \\
\hline 1 & 2 & 1 & 4.9 & 3 & 2 & 1 & 4.0 & 5 & 2 & 1 & 3.7 \\
\hline 1 & 2 & 2 & 5.1 & 3 & 2 & 2 & 4.1 & 5 & 2 & 2 & 3.7 \\
\hline 1 & 2 & 3 & 6.2 & 3 & 2 & 3 & 5.0 & 5 & 2 & 3 & 5.0 \\
\hline 1 & 2 & 4 & 6.8 & 3 & 2 & 4 & 5.6 & 5 & 2 & 4 & 5.8 \\
\hline 1 & 2 & 5 & 7.3 & 3 & 2 & 5 & 6.0 & 5 & 2 & 5 & 6.3 \\
\hline 1 & 3 & 1 & 5.1 & 3 & 3 & 1 & 3.8 & 5 & 3 & 1 & 3.7 \\
\hline 1 & 3 & 2 & 5.0 & 3 & 3 & 2 & 3.7 & 5 & 3 & 2 & 4.0 \\
\hline 1 & 3 & 3 & 5.8 & 3 & 3 & 3 & 4.9 & 5 & 3 & 3 & 4.8 \\
\hline 1 & 3 & 4 & 6.0 & 3 & 3 & 4 & 5.4 & 5 & 3 & 4 & 5.1 \\
\hline 1 & 3 & 5 & 7.0 & 3 & 3 & 5 & 5.6 & 5 & 3 & 5 & 5.5 \\
\hline 2 & 1 & 1 & 5.4 & 4 & 1 & 1 & 5.2 & 6 & 1 & 1 & 5.6 \\
\hline 2 & 1 & 2 & 5.8 & 4 & 1 & 2 & 5.7 & 6 & 1 & 2 & 5.9 \\
\hline 2 & 1 & 3 & 7.6 & 4 & 1 & 3 & 6.7 & 6 & 1 & 3 & 7.7 \\
\hline 2 & 1 & 4 & 8.3 & 4 & 1 & 4 & 7.7 & 6 & 1 & 4 & 7.8 \\
\hline 2 & 1 & 5 & 8.0 & 4 & 1 & 5 & 8.5 & 6 & 1 & 5 & 8.0 \\
\hline 2 & 2 & 1 & 3.9 & 4 & 2 & 1 & 4.0 & 6 & 2 & 1 & 3.8 \\
\hline 2 & 2 & 2 & 4.6 & 4 & 2 & 2 & 4.3 & 6 & 2 & 2 & 4.3 \\
\hline 2 & 2 & 3 & 5.0 & 4 & 2 & 3 & 5.2 & 6 & 2 & 3 & 5.4 \\
\hline 2 & 2 & 4 & 6.0 & 4 & 2 & 4 & 6.2 & 6 & 2 & 4 & 5.6 \\
\hline 2 & 2 & 5 & 6.3 & 4 & 2 & 5 & 6.4 & 6 & 2 & 5 & 6.3 \\
\hline 2 & 3 & 1 & 4.9 & 4 & 3 & 1 & 4.1 & 6 & 3 & 1 & 4.3 \\
\hline 2 & 3 & 2 & 4.8 & 4 & 3 & 2 & 4.1 & 6 & 3 & 2 & 4.3 \\
\hline 2 & 3 & 3 & 5.2 & 4 & 3 & 3 & 5.2 & 6 & 3 & 3 & 4.8 \\
\hline 2 & 3 & 4 & 6.0 & 4 & 3 & 4 & 5.7 & 6 & 3 & 4 & 5.9 \\
\hline 2 & 3 & 5 & 6.0 & 4 & 3 & 5 & 5.9 & 6 & 3 & 5 & 6.2 \\
\hline
\end{tabular}


The results presented in Tables 3 and 4 were obtained with the use of the empirical estimates (i.e., based on $\hat{\sigma}_{1}^{2}=0.05982222, \hat{\sigma}_{2}^{2}=0.1498667$ and $\left.\hat{\sigma}_{3}^{2}=1.955733\right)$

$$
\begin{aligned}
& \tilde{\boldsymbol{\tau}}=[5.300,5.700,7.050,7.933,8.217,4.050,4.350,5.300, \\
& 6.000,6.433,4.317,4.317,5.117,5.683,6.033]
\end{aligned}
$$

and

$$
\begin{aligned}
& \tilde{\boldsymbol{\tau}}_{*}=[-0.420,-0.020,1.330,2.213,2.497,-1.670,-1.370,-0.420, \\
& 0.280, \quad 0.713,-1.403,-1.403,-0.603,-0.037,0.313]^{\prime} \text {, }
\end{aligned}
$$

the former obtainable by the use of formula (8), the latter either from the relation $\tilde{\boldsymbol{\tau}}_{*}=\left(\boldsymbol{I}_{v}-n^{-1} \mathbf{1}_{v} \boldsymbol{r}^{\prime}\right) \tilde{\boldsymbol{\tau}}$, or directly by formula (32).

Table 3. Analysis of variance of the experiment in a split-plot design analyzed in Example 1

\begin{tabular}{lccccc}
\hline $\begin{array}{c}\text { Source } \\
\text { of variation }\end{array}$ & $\begin{array}{c}\text { Degrees } \\
\text { of freedom }\end{array}$ & $\begin{array}{c}\text { Sum } \\
\text { of squares }\end{array}$ & $\begin{array}{c}\text { Mean } \\
\text { square }\end{array}$ & $\hat{F}$ & $P$ value \\
\hline Treatments & 14 & 1728.1444 & 123.4389 & 123.4389 & $<0.0001$ \\
Residuals & 75 & 75 & 1 & - & - \\
\hline Total & 89 & 1803.1444 & - & - & - \\
\hline
\end{tabular}

Table 4. Analysis of variance for the sets of contrasts considered in Example 1

\begin{tabular}{cccccc}
\hline Source & $\begin{array}{c}\text { Degrees } \\
\text { of freedom }\end{array}$ & $\begin{array}{c}\text { Sum } \\
\text { of squares }\end{array}$ & $\begin{array}{c}\text { Mean } \\
\text { square }\end{array}$ & $\hat{F}$ & $P$ value \\
\hline Treatments & 14 & 1728.1444 & 123.4389 & 123.4389 & $<0.0001$ \\
A & 2 & 378.4342 & 189.2171 & 189.2171 & $<0.0001$ \\
B & 4 & 1288.7927 & 322.1982 & 322.1982 & $<0.0001$ \\
AB & 8 & 60.9175 & 7.6147 & 7.6147 & $<0.0001$ \\
Residuals & 75 & 75 & 1 & & \\
\hline Total & 89 & 1803.1444 & & & \\
\hline
\end{tabular}

Example 2. Mejza (1986) analyzed data from a plant breeding experiment carried out at the Dolne Pluczki Experimental Station. The aim of the experiment was to investigate the effect of nitrogen fertilization (factor A) on 
the tuber yield of nine medium-late potato varieties (factor B). The first experimental factor had three dose levels: A1 - 50 kg/ha, A2 - $100 \mathrm{~kg} / \mathrm{ha}$, and $\mathrm{A} 3-150 \mathrm{~kg} / \mathrm{ha}$. Due to the variability of soil fertility (environmental and soil conditions), it was appropriate to divide the experimental field into $b=18$ blocks of $60 \mathrm{~m}^{2}$ each. In each of the blocks, two main plots $\left(k_{1}=2\right)$ with $k_{2}=3$ sub-plots were separated due to the planned agrotechnical measures related to fertilization. Thus, $v=27$ treatments, as combinations of 3 levels of factor $\mathrm{A}$ and 9 levels of factor B, were distributed on $n=b k_{1} k_{2}=108$ experimental units (sub-plots) according to the incidence matrix $N=\left[N_{1}: N_{2}: \ldots .: N_{18}\right]$ in the following form, where the rows of the matrix correspond to individual treatment combinations:

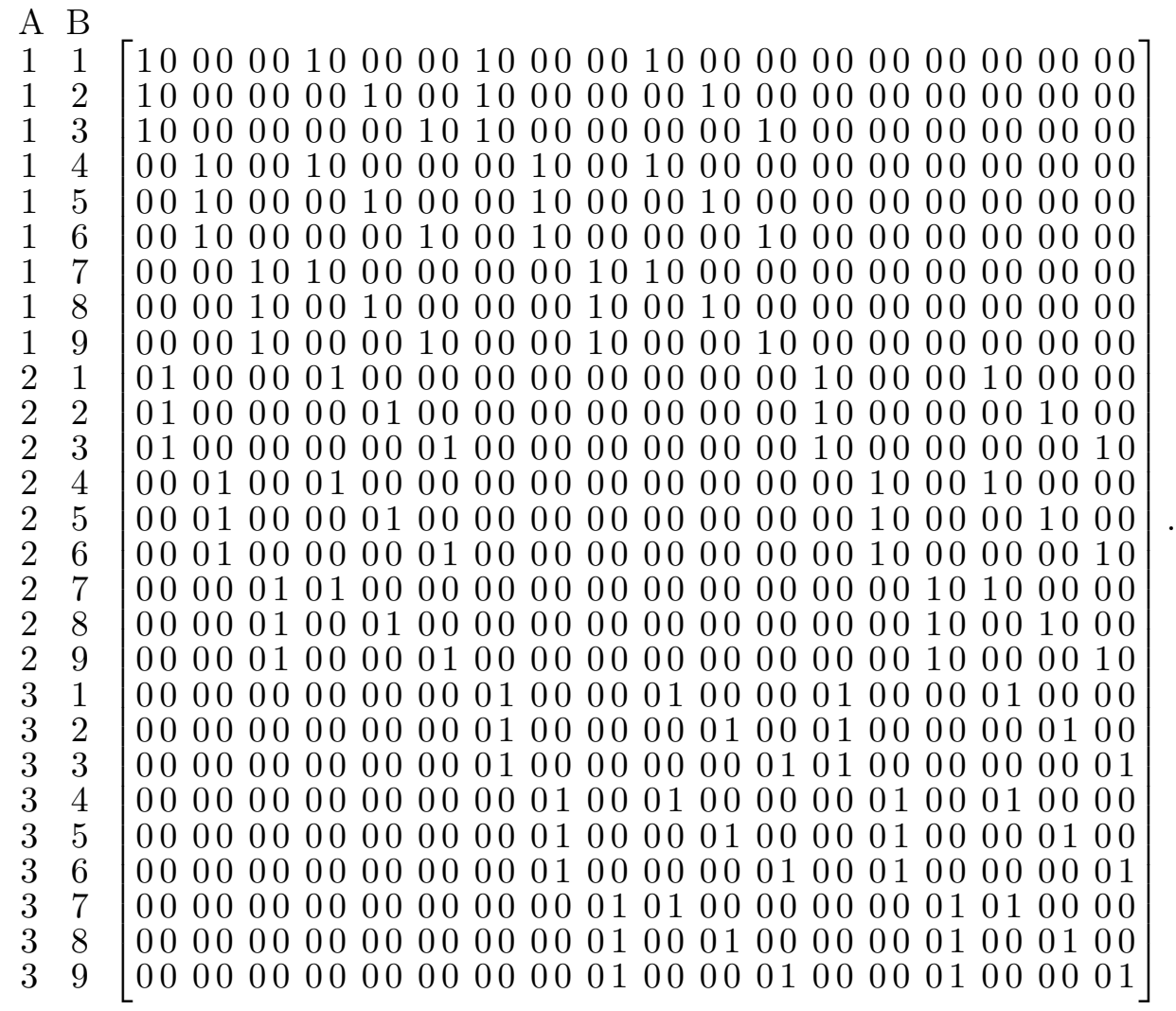

The above partition of the matrix $\boldsymbol{N}$ into the matrices $\boldsymbol{N}_{h}, h=1,2, \ldots, 18$, each composed of two columns, provides the design $\mathcal{D}$ described by the incidence matrix 


$$
\boldsymbol{M}=\left[\begin{array}{llllllllllllllllll}
1 & 0 & 0 & 1 & 0 & 0 & 1 & 0 & 0 & 1 & 0 & 0 & 0 & 0 & 0 & 0 & 0 & 0 \\
1 & 0 & 0 & 0 & 1 & 0 & 1 & 0 & 0 & 0 & 1 & 0 & 0 & 0 & 0 & 0 & 0 & 0 \\
1 & 0 & 0 & 0 & 0 & 1 & 1 & 0 & 0 & 0 & 0 & 1 & 0 & 0 & 0 & 0 & 0 & 0 \\
0 & 1 & 0 & 1 & 0 & 0 & 0 & 1 & 0 & 1 & 0 & 0 & 0 & 0 & 0 & 0 & 0 & 0 \\
0 & 1 & 0 & 0 & 1 & 0 & 0 & 1 & 0 & 0 & 1 & 0 & 0 & 0 & 0 & 0 & 0 & 0 \\
0 & 1 & 0 & 0 & 0 & 1 & 0 & 1 & 0 & 0 & 0 & 1 & 0 & 0 & 0 & 0 & 0 & 0 \\
0 & 0 & 1 & 1 & 0 & 0 & 0 & 0 & 1 & 1 & 0 & 0 & 0 & 0 & 0 & 0 & 0 & 0 \\
0 & 0 & 1 & 0 & 1 & 0 & 0 & 0 & 1 & 0 & 1 & 0 & 0 & 0 & 0 & 0 & 0 & 0 \\
0 & 0 & 1 & 0 & 0 & 1 & 0 & 0 & 1 & 0 & 0 & 1 & 0 & 0 & 0 & 0 & 0 & 0 \\
1 & 0 & 0 & 1 & 0 & 0 & 0 & 0 & 0 & 0 & 0 & 0 & 1 & 0 & 0 & 1 & 0 & 0 \\
1 & 0 & 0 & 0 & 1 & 0 & 0 & 0 & 0 & 0 & 0 & 0 & 1 & 0 & 0 & 0 & 1 & 0 \\
1 & 0 & 0 & 0 & 0 & 1 & 0 & 0 & 0 & 0 & 0 & 0 & 1 & 0 & 0 & 0 & 0 & 1 \\
0 & 1 & 0 & 1 & 0 & 0 & 0 & 0 & 0 & 0 & 0 & 0 & 0 & 1 & 0 & 1 & 0 & 0 \\
0 & 1 & 0 & 0 & 1 & 0 & 0 & 0 & 0 & 0 & 0 & 0 & 0 & 1 & 0 & 0 & 1 & 0 \\
0 & 1 & 0 & 0 & 0 & 1 & 0 & 0 & 0 & 0 & 0 & 0 & 0 & 1 & 0 & 0 & 0 & 1 \\
0 & 0 & 1 & 1 & 0 & 0 & 0 & 0 & 0 & 0 & 0 & 0 & 0 & 0 & 1 & 1 & 0 & 0 \\
0 & 0 & 1 & 0 & 1 & 0 & 0 & 0 & 0 & 0 & 0 & 0 & 0 & 0 & 1 & 0 & 1 & 0 \\
0 & 0 & 1 & 0 & 0 & 1 & 0 & 0 & 0 & 0 & 0 & 0 & 0 & 0 & 1 & 0 & 0 & 1 \\
0 & 0 & 0 & 0 & 0 & 0 & 1 & 0 & 0 & 1 & 0 & 0 & 1 & 0 & 0 & 1 & 0 & 0 \\
0 & 0 & 0 & 0 & 0 & 0 & 1 & 0 & 0 & 0 & 1 & 0 & 1 & 0 & 0 & 0 & 1 & 0 \\
0 & 0 & 0 & 0 & 0 & 0 & 1 & 0 & 0 & 0 & 0 & 1 & 1 & 0 & 0 & 0 & 0 & 1 \\
0 & 0 & 0 & 0 & 0 & 0 & 0 & 1 & 0 & 1 & 0 & 0 & 0 & 1 & 0 & 1 & 0 & 0 \\
0 & 0 & 0 & 0 & 0 & 0 & 0 & 1 & 0 & 0 & 1 & 0 & 0 & 1 & 0 & 0 & 1 & 0 \\
0 & 0 & 0 & 0 & 0 & 0 & 0 & 1 & 0 & 0 & 0 & 1 & 0 & 1 & 0 & 0 & 0 & 1 \\
0 & 0 & 0 & 0 & 0 & 0 & 0 & 0 & 1 & 1 & 0 & 0 & 0 & 0 & 1 & 1 & 0 & 0 \\
0 & 0 & 0 & 0 & 0 & 0 & 0 & 0 & 1 & 0 & 1 & 0 & 0 & 0 & 1 & 0 & 1 & 0 \\
0 & 0 & 0 & 0 & 0 & 0 & 0 & 0 & 1 & 0 & 0 & 1 & 0 & 0 & 1 & 0 & 0 & 1
\end{array}\right] .
$$

It is interesting to note that $\boldsymbol{M}=\boldsymbol{N}_{\mathrm{f}(\mathrm{A})} \otimes \boldsymbol{N}_{\mathrm{f}(\mathrm{B})}$, where

$$
\boldsymbol{N}_{\mathrm{f}(\mathrm{A})}=\left[\begin{array}{lll}
1 & 1 & 0 \\
1 & 0 & 1 \\
0 & 1 & 1
\end{array}\right] \quad \text { and } \quad \mathbf{N}_{\mathrm{f}(\mathrm{B})}=\left[\begin{array}{llllll}
1 & 0 & 0 & 1 & 0 & 0 \\
1 & 0 & 0 & 0 & 1 & 0 \\
1 & 0 & 0 & 0 & 0 & 1 \\
0 & 1 & 0 & 1 & 0 & 0 \\
0 & 1 & 0 & 0 & 1 & 0 \\
0 & 1 & 0 & 0 & 0 & 1 \\
0 & 0 & 1 & 1 & 0 & 0 \\
0 & 0 & 1 & 0 & 1 & 0 \\
0 & 0 & 1 & 0 & 0 & 1
\end{array}\right]
$$

the first defining the layout of the levels of factor $\mathrm{A}$, the second defining that of the levels of factor B.

It is assumed that the design used in the considered example was applied to available experimental units (sub-plots) grouped into main plots and those further into blocks, all of them constructed in such a way as to allow 
the appropriate threefold randomization to be performed, as indicated in Section 2.

The individual plot observations (plot yields) obtained for the combinations of the levels of factors A and B in the experiment considered in this example are presented in Table 5. The order of blocks in this table corresponds to the order of the columns of the incidence matrix given above.

When analyzing such data, the researcher is usually interested in estimating and testing certain sets of treatment parametric functions that can be defined as follows (assuming that the components of the vector $\boldsymbol{\tau}_{*}$ are ordered according to the order of the rows of the incidence matrix $\boldsymbol{N}$ given above):

$$
\begin{aligned}
& {\left[\left(\boldsymbol{I}_{3}-\frac{1}{3} \mathbf{1}_{3} \mathbf{1}_{3}^{\prime}\right) \otimes \frac{1}{9} \mathbf{1}_{9}^{\prime}\right] \boldsymbol{\tau}=\boldsymbol{U}_{\mathrm{A}}^{\prime} \boldsymbol{\tau} \equiv \boldsymbol{U}_{\mathrm{A}}^{\prime} \boldsymbol{\tau}_{*},} \\
& {\left[\frac{1}{3} \mathbf{1}_{3}^{\prime} \otimes\left(\boldsymbol{I}_{9}-\frac{1}{9} \mathbf{1}_{9} \mathbf{1}_{9}^{\prime}\right)\right] \boldsymbol{\tau}=\boldsymbol{U}_{\mathrm{B}}^{\prime} \boldsymbol{\tau} \equiv \boldsymbol{U}_{\mathrm{B}}^{\prime} \boldsymbol{\tau}_{*},} \\
& {\left[\left(\boldsymbol{I}_{3}-\frac{1}{3} \mathbf{1}_{3} \mathbf{1}_{3}^{\prime}\right) \otimes\left(\boldsymbol{I}_{9}-\frac{1}{9} \mathbf{1}_{9} \mathbf{1}_{9}^{\prime}\right)\right] \boldsymbol{\tau}=\boldsymbol{U}_{\mathrm{AB}}^{\prime} \boldsymbol{\tau} \equiv \boldsymbol{U}_{\mathrm{AB}}^{\prime} \boldsymbol{\tau}_{*},}
\end{aligned}
$$

where (46) stands for the main effects of the levels of factor A, (47) stands for the main effects of the levels of factor B, and (48) represents the interaction effects of these two factors. All these linear functions can be seen as contrasts of treatment parameters. For each of these three sets of contrasts, say $\boldsymbol{U}_{\mathrm{L}}$, the BLUE is obtainable according to formula (38), and the relevant sum of squares, $\operatorname{SS}\left(\boldsymbol{U}_{\mathrm{L}}\right)$, follows from (40).

It can be checked that the condition (44), $\boldsymbol{U}_{\mathrm{L}}^{\prime}\left(\boldsymbol{X}_{1}^{\prime} \boldsymbol{V}_{*}^{-1} \boldsymbol{X}_{1}\right)^{-1} \boldsymbol{U}_{\mathrm{L}^{*}}=\mathbf{O}$ for $\mathrm{L} \neq \mathrm{L}^{*}$, holds for any pair of the considered sets of contrasts, $\boldsymbol{U}_{\mathrm{A}}, \boldsymbol{U}_{\mathrm{B}}$ and $\boldsymbol{U}_{\mathrm{AB}}$, and then that the condition (42), extended to the three sets, in the form

$$
\begin{aligned}
& \left(\boldsymbol{X}_{1}^{\prime} \boldsymbol{V}_{*}^{-1} \boldsymbol{X}_{1}\right)^{-1} \boldsymbol{U}_{\mathrm{A}}\left[\boldsymbol{U}_{\mathrm{A}}^{\prime}\left(\boldsymbol{X}_{1}^{\prime} \boldsymbol{V}_{*}^{-1} \boldsymbol{X}_{1}\right)^{-1} \boldsymbol{U}_{\mathrm{A}}\right]^{-} \boldsymbol{U}_{\mathrm{A}}^{\prime} \\
& +\left(\boldsymbol{X}_{1}^{\prime} \boldsymbol{V}_{*}^{-1} \boldsymbol{X}_{1}\right)^{-1} \boldsymbol{U}_{\mathrm{B}}\left[\boldsymbol{U}_{\mathrm{B}}^{\prime}\left(\boldsymbol{X}_{1}^{\prime} \boldsymbol{V}_{*}^{-1} \boldsymbol{X}_{1}\right)^{-1} \boldsymbol{U}_{\mathrm{B}}\right]^{-} \boldsymbol{U}_{\mathrm{B}}^{\prime} \\
& +\left(\boldsymbol{X}_{1}^{\prime} \boldsymbol{V}_{*}^{-1} \boldsymbol{X}_{1}\right)^{-1} \boldsymbol{U}_{\mathrm{AB}}\left[\boldsymbol{U}_{\mathrm{AB}}^{\prime}\left(\boldsymbol{X}_{1}^{\prime} \boldsymbol{V}_{*}^{-1} \boldsymbol{X}_{1}\right)^{-1} \boldsymbol{U}_{\mathrm{AB}}\right]^{-} \boldsymbol{U}_{\mathrm{AB}}^{\prime} \\
& =\boldsymbol{I}_{v}-v^{-1} \mathbf{1}_{v} \mathbf{1}_{v}^{\prime},
\end{aligned}
$$

also holds.

This implies that

$$
\mathrm{SS}\left(\boldsymbol{U}_{\mathrm{A}}\right)+\mathrm{SS}\left(\boldsymbol{U}_{\mathrm{B}}\right)+\mathrm{SS}\left(\boldsymbol{U}_{\mathrm{AB}}\right)=\mathrm{SS}_{V},
$$


Table 5. Experimental observations of the field plot yield of the combinations of levels of the two factors analyzed in Example 2

\begin{tabular}{cccccccccccc}
\hline Block & A & B & Observ. & Block & A & B & Observ. & Block & A & B & Observ. \\
\hline 1 & 1 & 1 & 35.8 & 7 & 1 & 1 & 38.4 & 13 & 2 & 1 & 35.3 \\
1 & 1 & 2 & 48.3 & 7 & 1 & 2 & 49.1 & 13 & 2 & 2 & 41.2 \\
1 & 1 & 3 & 30.8 & 7 & 1 & 3 & 36.3 & 13 & 2 & 3 & 47.5 \\
1 & 2 & 1 & 28.5 & 7 & 3 & 1 & 40.2 & 13 & 3 & 1 & 38.0 \\
1 & 2 & 2 & 36.0 & 7 & 3 & 2 & 50.6 & 13 & 3 & 2 & 57.1 \\
1 & 2 & 3 & 37.0 & 7 & 3 & 3 & 47.5 & 13 & 3 & 3 & 43.4 \\
2 & 1 & 4 & 44.9 & 8 & 1 & 4 & 44.5 & 14 & 2 & 4 & 42.8 \\
2 & 1 & 5 & 40.8 & 8 & 1 & 5 & 46.2 & 14 & 2 & 5 & 48.0 \\
2 & 1 & 6 & 46.2 & 8 & 1 & 6 & 52.2 & 14 & 2 & 6 & 56.2 \\
2 & 2 & 4 & 46.0 & 8 & 3 & 4 & 52.0 & 14 & 3 & 4 & 47.0 \\
2 & 2 & 5 & 49.0 & 8 & 3 & 5 & 49.7 & 14 & 3 & 5 & 45.9 \\
2 & 2 & 6 & 47.5 & 8 & 3 & 6 & 60.3 & 14 & 3 & 6 & 54.9 \\
3 & 1 & 7 & 48.8 & 9 & 1 & 7 & 52.0 & 15 & 2 & 7 & 43.3 \\
3 & 1 & 8 & 46.0 & 9 & 1 & 8 & 55.8 & 15 & 2 & 8 & 50.8 \\
3 & 1 & 9 & 31.1 & 9 & 1 & 9 & 29.6 & 15 & 2 & 9 & 42.0 \\
3 & 2 & 7 & 44.6 & 9 & 3 & 7 & 57.0 & 15 & 3 & 7 & 53.8 \\
3 & 2 & 8 & 54.6 & 9 & 3 & 8 & 45.6 & 15 & 3 & 8 & 52.7 \\
3 & 2 & 9 & 37.3 & 9 & 3 & 9 & 50.3 & 15 & 3 & 9 & 47.3 \\
4 & 1 & 1 & 33.0 & 10 & 1 & 1 & 36.6 & 16 & 2 & 1 & 36.6 \\
4 & 1 & 4 & 46.1 & 10 & 1 & 4 & 43.5 & 16 & 2 & 4 & 42.6 \\
4 & 1 & 7 & 45.0 & 10 & 1 & 7 & 48.7 & 16 & 2 & 7 & 45.6 \\
4 & 2 & 1 & 30.2 & 10 & 3 & 1 & 41.5 & 16 & 3 & 1 & 45.0 \\
4 & 2 & 4 & 50.5 & 10 & 3 & 4 & 46.6 & 16 & 3 & 4 & 51.4 \\
4 & 2 & 7 & 47.6 & 10 & 3 & 7 & 51.6 & 16 & 3 & 7 & 57.1 \\
5 & 1 & 2 & 49.5 & 11 & 1 & 2 & 46.6 & 17 & 2 & 2 & 46.3 \\
5 & 1 & 5 & 42.8 & 11 & 1 & 5 & 40.2 & 17 & 2 & 5 & 47.0 \\
5 & 1 & 8 & 52.4 & 11 & 1 & 8 & 48.6 & 17 & 2 & 8 & 55.2 \\
5 & 2 & 2 & 38.5 & 11 & 3 & 2 & 53.3 & 17 & 3 & 2 & 57.5 \\
5 & 2 & 5 & 48.7 & 11 & 3 & 5 & 44.8 & 17 & 3 & 5 & 46.2 \\
5 & 2 & 8 & 52.8 & 11 & 3 & 8 & 50.3 & 17 & 3 & 8 & 51.0 \\
6 & 1 & 3 & 30.7 & 12 & 1 & 3 & 35.2 & 18 & 2 & 3 & 45.6 \\
6 & 1 & 6 & 50.2 & 12 & 1 & 6 & 47.8 & 18 & 2 & 6 & 53.4 \\
6 & 1 & 9 & 32.0 & 12 & 1 & 9 & 34.6 & 18 & 2 & 9 & 41.5 \\
6 & 2 & 3 & 43.8 & 12 & 3 & 3 & 45.4 & 18 & 3 & 3 & 49.0 \\
6 & 2 & 6 & 52.2 & 12 & 3 & 6 & 59.7 & 18 & 3 & 6 & 55.6 \\
6 & 2 & 9 & 44.0 & 12 & 3 & 9 & 45.0 & 18 & 3 & 9 & 48.4 \\
\hline & & & & & & & & &
\end{tabular}


with each component on the left in (50) obtainable using formula (40), with $\hat{\boldsymbol{\tau}}_{*}$ obtained by the use of formula (32). With these conditions satisfied, one can proceed to the general ANOVA and to its partition into three components related to the three sets of contrasts.

Table 6. Analysis of variance for the experiment in a split-plot design analyzed in Example 2

\begin{tabular}{lccc}
\hline $\begin{array}{c}\text { Source } \\
\text { of variation }\end{array}$ & $\begin{array}{c}\text { Degrees } \\
\text { of freedom }\end{array}$ & $\begin{array}{c}\text { Sum } \\
\text { of squares }\end{array}$ & $\begin{array}{c}\text { Mean } \\
\text { square }\end{array}$ \\
\hline Treatments & 26 & 590.7361 & 22.7206 \\
Residuals & 81 & 81 & 1 \\
\hline Total & 107 & 671.7361 & - \\
\hline
\end{tabular}

Table 7. Analysis of variance for the sets of contrasts considered in Example 2

\begin{tabular}{cccccc}
\hline Source & $\begin{array}{c}\text { Degrees } \\
\text { of freedom }\end{array}$ & $\begin{array}{c}\text { Sum } \\
\text { of squares }\end{array}$ & $\begin{array}{c}\text { Mean } \\
\text { square }\end{array}$ & $\hat{F}$ & $P$ value \\
\hline Treatments & 26 & 590.7361 & 22.7206 & 22.7206 & $<0.0001$ \\
A & 2 & 89.7859 & 44.8930 & 44.8930 & $<0.0001$ \\
B & 8 & 367.3033 & 45.9129 & 45.9129 & $<0.0001$ \\
AB & 16 & 133.6469 & 8.3529 & 8.3529 & $<0.0001$ \\
Residuals & 81 & 81 & 1 & & \\
\hline Total & 107 & 671.7361 & & & \\
\hline
\end{tabular}

The critical values, at the 1 percent level of significance, for the approximate distribution of the above test statistic $\hat{F}$, following from (29), are: 1.76 for 26 d.f., 4.61 for 2 d.f., 2.51 for 8 d.f. and 2.00 for 16 d.f.

The results presented in Tables 6 and 7 were obtained with the use of the empirical estimates (i.e., based on $\hat{\sigma}_{1}^{2}=6.904256, \hat{\sigma}_{2}^{2}=8.792828$ and $\left.\hat{\sigma}_{3}^{2}=13.68151\right)$

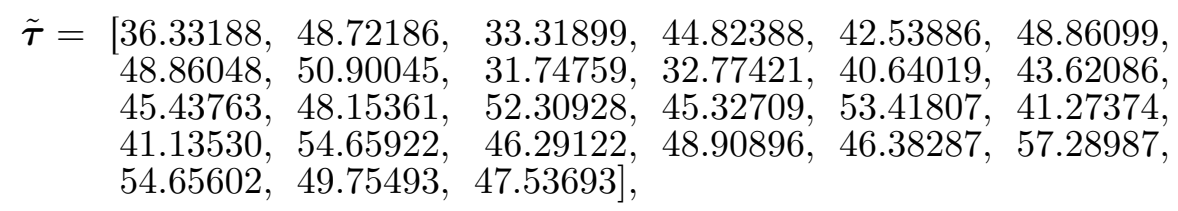

and 


$$
\begin{aligned}
& \tilde{\boldsymbol{\tau}}_{*}=[-9.4338589,2.9561158,-12.4467470,-0.9418603,-3.2268856, \\
& 3.0952516, \quad 3.0947354, \quad 5.1347101,-14.0181527,-12.9915269 \text {, } \\
& -5.1255488,-2.1448806,-0.3281064, \quad 2.3878718,6.5435400 \text {, } \\
& -0.4386462, \quad 7.6523319,-4.4919999,-4.6304407, \quad 8.8934766 \text {, } \\
& 0.5254767, \quad 3.1432154, \quad 0.6171327, \quad 11.5241327, \quad 8.8902762 \text {, } \\
& \text { 3.9891935, 1.7711935], }
\end{aligned}
$$

the former obtainable by the use of formula (8), the latter either from the relation $\tilde{\boldsymbol{\tau}}_{*}=\left(\boldsymbol{I}_{v}-n^{-1} \mathbf{1}_{v} \boldsymbol{r}^{\prime}\right) \tilde{\boldsymbol{\tau}}$, or directly by formula (32). Using $\tilde{\boldsymbol{\tau}}_{*}$ in the formulae (34) and (40), i.e., replacing $\boldsymbol{V}_{*}^{-1}$ by $\hat{\boldsymbol{V}}_{*}^{-1}$ there, the estimated sum of squares $\widehat{\mathrm{SS}}_{V}$ and its relevant components are obtained. Evidently, as follows from (28), the estimated residual sum of squares $\widehat{\mathrm{SS}}_{R}$ is reduced to $n-v$, its d.f.

\section{Concluding remarks}

The present paper is the fourth in the planned series concerning a new approach to the analysis of experiments with the OBS property. The first paper in this series (Caliński and Siatkowski, 2017) concerns experiments conducted in proper block designs. The second (Caliński and Siatkowski, 2018) is devoted to experiments in nested block designs. The third (Caliński, Łacka and Siatkowski, 2019) concerns experiments in row-column designs. Here the new approach is applied to experiments in split-plot designs that induce the OBS property.

Exactly as in the first three works, it appears that when the unknown stratum variances within the covariance (dispersion) matrix $\boldsymbol{V}$, given in (3), are replaced by their estimates, obtained from the estimation procedure suggested by Nelder (1968), the residual sum of squares $\mathrm{SS}_{R}$ is reduced to its d.f., that is, its expectation. This result is obtainable due to the proposed new approach to the analysis of experimental data.

Another feature of the proposed approach relates to simplification of the main analytical procedures, as presented in Section 4. One of the resulting advantages is the reduction of the number of stratum variances involved, which substantially simplifies the computations.

However, as can be seen from the examples analyzed in Section 6, the main advantage of the proposed approach is the fact that the ANOVA results are obtainable directly, not by first performing some partial analyses, under relevant stratum submodels, and then combining their results (as suggested in most of the relevant literature). 
The indicated advantages are similar to those presented in the previous three papers in this series, planned for different classes of designs inducing the OBS property.

\section{REFERENCES}

Bailey, R. A. (2008): Design of Comparative Experiments. Cambridge University Press.

Brzeskwiniewicz H. (1994): Experiment with split-plot generated by PBIB designs. Biometrical Journal 36: 557-570.

Caliński T., Kageyama S. (2000): Block Designs: A Randomization Approach, Vol. I: Analysis. Lecture Notes in Statistics 150, Springer, New York.

Caliński T., Siatkowski I. (2017): On a new approach to the analysis of variance for experiments with orthogonal block structure. I. Experiments in proper block designs. Biometrical Letters 54: 91-122.

Caliński T., Siatkowski I. (2018): On a new approach to the analysis of variance for experiments with orthogonal block structure. II. Experiments in nested block designs. Biometrical Letters 55: 147-178.

Caliński T., Łacka A., Siatkowski I. (2019): On a new approach to the analysis of variance for experiments with orthogonal block structure. III. Experiments in row-column designs. Biometrical Letters 56: 183-213.

Elandt R. (1964): Statystyka matematyczna w zastosowaniu do doświadczalnictwa rolniczego. Państwowe Wydawnictwo Naukowe, Warszawa, Poland.

Hinkelmann K., Kempthorne O. (2008): Design and Analysis of Experiments, Vol. 1: Introduction to Experimental Design, 2nd ed. Wiley, Hoboken, New Jersey.

Houtman A.M., Speed T.P. (1983): Balance in designed experiments with orthogonal block structure. Annals of Statistics 11: 1069-1085.

Johnson N.L., Kotz S., Balakrishnan N. (1995): Continuous Univariate Distributions, Vol. 2, 2nd ed. Wiley, New York.

Kala R. (2019): A new look at combining information from stratum submodels. In: Ahmed S., Carvalho F., Puntanen S. (eds.) Matrices, Statistics and Big Data. IWMS 2016. Contributions to Statistics. Springer: 35-49.

Mejza I. (1996): Control treatments in incomplete split-plot designs. Tatra Mountains Mathematical Publications 7: 69-77.

Mejza S. (1986): Doświadczenia w układach blokowych niekompletnych o jednostkach rozszczepionych. Rozprawy Naukowe 150, Roczniki Akademii Rolniczej w Poznaniu.

Nelder J.A. (1965): The analysis of randomized experiments with orthogonal block structure. Proceedings of the Royal Society of London, Series A 283: 147-178.

Nelder J.A. (1968): The combination of information in generally balanced designs. Journal of the Royal Statistical Society, Series B 30: 303-311.

Pearce S.C. (1983): The Agricultural Field Experiment: A Statistical Examination of Theory and Practice. Wiley, New York. 
Rao C.R. (1971): Unified theory of linear estimation. Sankhyā, Series A 33: 371394.

Rao C.R. (1974): Projectors, generalized inverses and the BLUE's. Journal of the Royal Statistical Society, Series B 36: 442-448.

Rao C.R., Mitra S.K. (1971): Generalized Inverse of Matrices and its Applications. Wiley, New York.

R Core Team (2017): R: A language and environment for statistical computing. R Foundation for Statistical Computing, Vienna, Austria. URL https://www.Rproject.org/.

Volaufova J. (2009): Heteroscedastic ANOVA: old $p$ values, new views. Statistical Papers 50: 943-962.

Yates F. (1933): The principles of orthogonality and confounding in replicated experiments. Journal of Agricultural Science 23: 108-145.

Yates F. (1935): Complex experiments. Supplement to the Journal of the Royal Statistical Society 2: 181-247.

Yates F. (1939): The recovery of inter-block information in variety trials arranged in three-dimensional lattices. Annals of Eugenics 9: 136-156.

Yates F. (1940): The recovery of inter-block information in balanced incomplete block designs. Annals of Eugenics 10: 317-325. 